\title{
A Nonparametric Model of Random Uncertainties for Reduced Matrix Models in Structural Dynamics
}

\author{
C. Soize \\ Structural Dynamics and Coupled Systems Department \\ Office National d'Études et de Recherches Aérospatiales (ONERA) \\ BP 72, 92322 Chatillon Cedex, France
}

\begin{abstract}
Random uncertainties in finite element models in linear structural dynamics are usually modeled by using parametric models. This means that 1) the uncertain local parameters occurring in the global mass, damping and stiffness matrices of the finite element model have to be identified; 2) appropriate probabilistic models of these uncertain parameters have to be constructed, and 3) functions mapping the domains of uncertain parameters into the global mass, damping and stiffness matrices have to be constructed. In the low-frequency range, a reduced matrix model can then be constructed using the generalized coordinates associated with the structural modes corresponding to the lowest eigenfrequencies. In this paper we propose an approach for constructing a random uncertainties model of the generalized mass, damping and stiffness matrices. This nonparametric model does not require identifying the uncertain local parameters and consequently, obviates construction of functions which map the domains of uncertain local parameters into the generalized mass, damping and stiffness matrices. This nonparametric model of random uncertainties is based on direct construction of a probabilistic model of the generalized mass, damping and stiffness matrices, which uses only the available information constituted of the mean value of the generalized mass, damping and stiffness matrices. This paper describes the explicit construction of the theory of such a nonparametric model.
\end{abstract}

Keywords: Structural dynamics; random uncertainties; finite element model; entropy optimization principle

\section{Introduction}

\subsection{Introduction of the "mean finite element model"}

Let us consider the finite element model of linear vibrations of a damped structure around a position of static equilibrium taken as reference configuration without prestresses. Details concerning such a model can be found in the literature on structural dynamics and vibrations [1-6]. In this paper, we are interested in predicting the frequency response functions of such a system in the frequency band of analysis

$$
\mathbb{B}=\left[\omega_{\min }, \omega_{\max }\right] \quad, \quad 0<\omega_{\min }<\omega_{\max } .
$$

With respect to frequency band $\mathbb{B}$ of analysis, it is assumed that the structure under consideration can be modeled by a linear time-invariant damped dynamical system. Consequently, this finite element model, which has to be considered as the "mean finite element model" can be written in the time domain as

$$
[\underline{M}] \underline{\ddot{\mathbf{y}}}(t)+[\underline{D}] \underline{\dot{\mathbf{y}}}(t)+[\underline{K}] \underline{\mathbf{y}}(t)=\mathbf{x}(t)
$$

or in the frequency domain as

$$
\left(-\omega^{2}[\underline{M}]+i \omega[\underline{D}]+[\underline{K}]\right) \underline{\mathbf{y}}(\omega)=\mathbf{x}(\omega)
$$

in which $\underline{\mathbf{y}}=\left(\underline{y}_{1}, \ldots, \underline{y}_{m}\right)$ is the vector of the $m$ DOFs (displacements and/or rotations) and $\mathbf{x}=\left(x_{1}, \ldots, x_{m}\right)$ is the vector of the $m$ inputs (forces and/or moments). The mass, damping and stiffness matrices $[\underline{M}],[\underline{D}]$ and 
$[\underline{K}]$ are symmetric $(m \times m)$ real matrices independent of $\omega$ over band $\mathbb{B}$, but may depend on $\mathbb{B}$ (as is the case if $\mathbb{B}$ is a narrow medium-frequency band and if the structure is modeled with viscoelastic constitutive equations [6]). Matrix $[\underline{M}]$ is positive definite whereas matrices $[\underline{D}]$ and $[\underline{K}]$ are either positive definite (fixed structure) or positive semidefinite if there are rigid body modes (free structure). In the case of a free structure, the external forces are assumed to be in equilibrium in the sense defined below. It is assumed that matrices $[\underline{D}]$ and $[\underline{K}]$ have the same null space. Note that an underlined quantity refers to the "mean finite element model". For all $\omega$ fixed in $\mathbb{B}$, Eq. (2) has a unique solution $\underline{\mathbf{y}}(\omega)=[\underline{h}(\omega)] \mathbf{x}(\omega)$ in which $\omega \mapsto[\underline{h}(\omega)]$ is the frequency response function defined on $\mathbb{B}$ with values in the symmetric $(m \times m)$ complex matrices,

$$
[\underline{h}(\omega)]=\left(-\omega^{2}[\underline{M}]+i \omega[\underline{D}]+[\underline{K}]\right)^{-1} .
$$

\subsection{Introduction of the "mean reduced matrix model"}

If frequency band $\mathbb{B}$ corresponds to the low-frequency range, a reduced matrix model can be constructed using the structural modes $[1,2,4-6]$. Let us consider the generalized eigenvalue problem related to the mean finite element model,

$$
[\underline{K}] \mathbf{y}=\lambda[\underline{M}] \mathbf{y}
$$

1) If the structure is fixed, then $[\underline{K}]$ and $[\underline{D}]$ are positive-definite matrices. We have $0<\lambda_{1} \leq \lambda_{2} \leq \ldots$ and the associated eigenvectors $\left\{\mathbf{y}_{1}, \mathbf{y}_{2}, \ldots\right\}$ are such that

$$
<[\underline{M}] \mathbf{y}_{\alpha}, \mathbf{y}_{\beta}>=\delta_{\alpha \beta} \quad, \quad<[\underline{K}] \mathbf{y}_{\alpha}, \mathbf{y}_{\beta}>=\omega_{\alpha}^{2} \delta_{\alpha \beta} \quad,
$$

in which $\omega_{\alpha}=\sqrt{\lambda_{\alpha}}$ is the eigenfrequency of structural mode $\mathbf{y}_{\alpha}$ whose normalization is defined by generalized mass $\mu_{\alpha}=1$ and where $<\mathbf{y}, \mathbf{x}>=y_{1} x_{1}+\ldots+y_{m} x_{m}$.

2) If the structure is free, then $[\underline{K}]$ and $[\underline{D}]$ are positive-semidefinite matrices. The dimension of the null space of $[\underline{K}]$ is denoted as $m_{\text {rig }}$ and is assumed to be such that $0<m_{\text {rig }} \leq 6$. The rigid body modes are denoted as $\left\{\mathbf{y}_{-m_{\text {rig }}}, \ldots, \mathbf{y}_{-1}\right\}$ and verify $[\underline{K}] \mathbf{y}_{\alpha}=0$ for $\alpha$ in $\left\{-m_{\text {rig }}, \ldots,-1\right\}$. Since by assumption the null spaces of matrices $[\underline{D}]$ and $[\underline{K}]$ coincide, the null space of $[\underline{D}]$ is spanned by $\left\{\mathbf{y}_{-m_{\text {rig }}}, \ldots, \mathbf{y}_{-1}\right\}$. The structural modes $\left\{\mathbf{y}_{1}, \mathbf{y}_{2}, \ldots\right\}$ are associated with positive eigenvalues $0<\lambda_{1} \leq \lambda_{2} \leq \ldots$. Vectors $\mathbf{y}_{\alpha}$ belonging to the family $\left\{\mathbf{y}_{-m_{\text {rig }}}, \ldots, \mathbf{y}_{-1}, \mathbf{y}_{1}, \mathbf{y}_{2}, \ldots\right\}$ constituted of the rigid body modes and the structural modes verify the orthogonality properties defined by Eq. (5). Since external force $\mathbf{x}(\omega)$ is in equilibrium, for all $\omega$ in $\mathbb{B}$, we have

$$
<\mathbf{x}(\omega), \mathbf{y}_{\alpha}>=0 \quad, \quad \alpha \in\left\{-m_{\text {rig }}, \ldots,-1\right\} .
$$

3) The mean reduced matrix model related to structural modes $\left\{\mathbf{y}_{1}, \ldots, \mathbf{y}_{N}\right\}$ with $N \ll m$ is then defined by the frequency response function $\left[\underline{h}_{N}(\omega)\right]$ such that

$$
\left[\underline{h}_{N}(\omega)\right]=\sum_{\alpha=1}^{N} \sum_{\beta=1}^{N}[\underline{\mathcal{T}}(\omega)]_{\alpha \beta} \mathbf{y}_{\alpha} \mathbf{y}_{\beta}^{T},
$$

in which $[\underline{\mathcal{I}}(\omega)]$ is the $(N \times N)$ complex symmetric matrix defined by

$$
[\underline{\mathcal{I}}(\omega)]=\left(-\omega^{2}[\underline{\mathcal{M}}]+i \omega[\underline{\mathcal{D}}]+[\underline{\mathcal{K}}]\right)^{-1},
$$

where $[\underline{\mathcal{M}}],[\underline{\mathcal{D}}]$ and $[\underline{\mathcal{K}}]$ are the mean generalized mass, damping and stiffness matrices which are symmetric positive-definite $(N \times N)$ real matrices such that $[\underline{\mathcal{M}}]_{\alpha \beta}=\delta_{\alpha \beta},[\underline{\mathcal{D}}]_{\alpha \beta}=<[\underline{D}] \mathbf{y}_{\beta}, \mathbf{y}_{\alpha}>$ and $[\underline{\mathcal{K}}]_{\alpha \beta}=\omega_{\alpha}^{2} \delta_{\alpha \beta}$. A quasi-static correction term can be added to the right-hand side of Eq. (6) to accelerate convergence [6].

4) It should be noted that the usual construction for the low-frequency range recalled above cannot be used if frequency band $\mathbb{B}$ belongs to the medium-frequency range. A mean reduced matrix model was recently proposed for the medium-frequency range [7-6]. This method consists in replacing the structural modes associated with the 
$N$ lowest eigenfrequencies of the mean finite element model by the eigenfunctions associated with the $N$ highest eigenvalues of the mechanical energy operator of the structure over band $\mathbb{B}$.

\subsection{The usual parametric model of random uncertainties in linear structural dynamics}

Random uncertainties are mainly due to uncertainties on the geometry, boundary conditions and constitutive equations. In this case, the matrices of the finite element model depend on a vector-valued uncertain parameter denoted as $\underline{\mathbf{q}}+\mathbf{q}$ in which $\underline{\mathbf{q}}=\left(\underline{q}_{1}, \ldots, \underline{q}_{L}\right) \in \mathbb{R}^{L}$ is the mean value of the uncertain parameter and $\mathbf{q}$ is the fluctuation around mean value $\underline{\mathbf{q}}$. Fluctuation $\mathbf{q}=\left(q_{1}, \ldots, q_{L}\right)$ is such that $\mathbf{q} \in \mathcal{D}_{L} \subset \mathbb{R}^{L}$, where $\mathcal{D}_{L}$ is a subspace of $\mathbb{R}^{L}$. Consequently, Eq. (2) is rewritten as

$$
\left(-\omega^{2}[M(\underline{\mathbf{q}}+\mathbf{q})]+i \omega[D(\underline{\mathbf{q}}+\mathbf{q})]+[K(\underline{\mathbf{q}}+\mathbf{q})]\right) \mathbf{y}(\omega)=\mathbf{x}(\omega) \quad .
$$

If $\mathbf{q}=0$, this equation yields the "mean finite element model" defined by Eq. (2) with $[\underline{M}]=[M(\underline{\mathbf{q}})]$, $[\underline{D}]=[D(\underline{\mathbf{q}})]$ and $[\underline{K}]=[K(\underline{\mathbf{q}})]$. Matrix $[M(\underline{\mathbf{q}}+\mathbf{q})]$ is positive definite and it is assumed that the null spaces of matrices $[D(\underline{\mathbf{q}}+\mathbf{q})]$ and $[K(\underline{\mathbf{q}}+\mathbf{q})]$ are independent of $\mathbf{q}$ and coincide with the null space of $[\underline{K}]$. Uncertain parameter $\mathbf{q} \in \overline{\mathcal{D}}_{L} \subset \mathbb{R}^{L}$ is modeled by a second-order centered random variable $\mathbf{Q}=\left(Q_{1}, \ldots, Q_{L}\right)$ with valued in $\mathbb{R}^{L}$, whose probability distribution is written as $P_{\mathbf{Q}}(d \mathbf{q})=p_{\mathbf{Q}}(\mathbf{q}) d \mathbf{q}$ in which $p_{\mathbf{Q}}(\mathbf{q})=0$ if $\mathbf{q} \notin \mathcal{D}_{L}$. In practice, functions $\mathbf{q} \mapsto[M(\underline{\mathbf{q}}+\mathbf{q})],[D(\underline{\mathbf{q}}+\mathbf{q})]$ and $[K(\underline{\mathbf{q}}+\mathbf{q})]$ have to be algebraically known and in the general case, only a numerical approximation can be constructed using a sensitivity analysis of the finite element model with respect to uncertain parameter q. Such a sensitivity analysis can be performed using a first- or second-order analysis. Consequently, in the frequency domain, the response of the dynamical system is a random vector $\mathbf{Y}(\omega)$ with values in $\mathbb{C}^{m}$ verifying the random matrix equation

$$
\left(-\omega^{2}[M(\underline{\mathbf{q}}+\mathbf{Q})]+i \omega[D(\underline{\mathbf{q}}+\mathbf{Q})]+[K(\underline{\mathbf{q}}+\mathbf{Q})]\right) \mathbf{Y}(\omega)=\mathbf{x}(\omega) \quad .
$$

For all $\omega$ fixed in $\mathbb{B}$, this equation has a unique solution $\mathbf{Y}(\omega)=[\mathbf{H}(\omega)] \mathbf{x}(\omega)$ in which $[\mathbf{H}(\omega)]$ is a random matrix with values in the set of the symmetric $(m \times m)$ complex matrices and is defined by $[\mathbf{H}(\omega)]=[h(\omega, \mathbf{Q})]$ in which

$$
[h(\omega, \mathbf{q})]=\left(-\omega^{2}[M(\underline{\mathbf{q}}+\mathbf{q})]+i \omega[D(\underline{\mathbf{q}}+\mathbf{q})]+[K(\underline{\mathbf{q}}+\mathbf{q})]\right)^{-1} .
$$

Any probabilistic quantity related to stochastic process $\{\mathbf{Y}(\omega), \omega \in \mathbb{B}\}$ can be constructed using the above model. For instance, the mean value of random vector $\mathbf{Y}(\omega)$ is given by $E\{\mathbf{Y}(\omega)\}=E\{[\mathbf{H}(\omega)]\} \mathbf{x}(\omega)$ in which

$$
E\{[\mathbf{H}(\omega)]\}=\int_{\mathcal{D}_{L}}[h(\omega, \mathbf{q})] p_{\mathbf{Q}}(\mathbf{q}) d \mathbf{q}
$$

It should be noted that mean value $E\{[\mathbf{H}(\omega)]\}$ is not equal to frequency response function $[\underline{h}(\omega)]$ related to the "mean finite element model". In practice, the $L$-uple integral over $\mathcal{D}_{L}$ can be estimated, either using a firstor second-order Taylor expansion of function $\mathbf{q} \mapsto[h(\omega, \mathbf{q})]$, or using a Monte Carlo calculation of the $L$-uple integral.

\subsubsection{Random reduced matrix model}

The reduced matrix model related to structural modes $\left\{\mathbf{y}_{1}, \ldots, \mathbf{y}_{N}\right\}$ introduced in Section 1.1.1 with $N \ll m$ consists in replacing frequency response function $[h(\omega, \mathbf{q})]$ by its approximation

$$
\left[h_{N}(\omega, \mathbf{q})\right]=\sum_{\alpha=1}^{N} \sum_{\beta=1}^{N}[\mathcal{T}(\omega, \mathbf{q})]_{\alpha \beta} \mathbf{y}_{\alpha} \mathbf{y}_{\beta}^{T}
$$

in which $\left[h_{N}(\omega, \mathbf{q})\right]$ is a symmetric $(m \times m)$ complex matrix and $[\mathcal{T}(\omega, \mathbf{q})]$ is a symmetric $(N \times N)$ complex matrix such that

$$
[\mathcal{T}(\omega, \mathbf{q})]=\left(-\omega^{2}[\mathcal{M}(\underline{\mathbf{q}}+\mathbf{q})]+i \omega[\mathcal{D}(\underline{\mathbf{q}}+\mathbf{q})]+[\mathcal{K}(\underline{\mathbf{q}}+\mathbf{q})]\right)^{-1}
$$


where generalized mass, damping and stiffness matrices $[\mathcal{M}(\mathbf{q}+\mathbf{q})],[\mathcal{D}(\underline{\mathbf{q}}+\mathbf{q})]$ and $[\mathcal{K}(\underline{\mathbf{q}}+\mathbf{q})]$ are symmetric positive-definite $(N \times N)$ real dense matrices which can be constructed explicitly using a first- or second-order sensitivity analysis to represent functions $\mathbf{q} \mapsto[M(\underline{\mathbf{q}}+\mathbf{q})],[D(\mathbf{q}+\mathbf{q})]$ and $[K(\mathbf{q}+\mathbf{q})]$. The random reduced matrix model related to structural modes $\left\{\mathbf{y}_{1}, \ldots, \mathbf{y}_{N}\right\}$ with $N \ll m$ is then defined by

$$
\left[\mathbf{H}_{N}(\omega)\right]=\left[h_{N}(\omega, \mathbf{Q})\right] .
$$

The methods used for $[\mathbf{H}(\omega)]$ can be used to calculate the probabilistic quantities related to random frequency response function $\left[\mathbf{H}_{N}(\omega)\right]$ replacing $[h(\omega, \mathbf{q})]$ by $\left[h_{N}(\omega, \mathbf{q})\right]$.

A reduced matrix model can be obtained for the medium-frequency range using the above method, replacing the structural modes of the "mean finite element model" associated with the $N$ lowest eigenfrequencies by the eigenfunctions associated with the $N$ highest eigenvalues of the mechanical energy operator of the structure (mean finite element model) over band $\mathbb{B}$.

\subsubsection{Parametric model and bibliography}

The approach described in Section 1.3.1 can be defined as a parametric random reduced matrix model because 1) uncertain vector parameter $\mathbf{q}$ is constituted of the local parameters of the mean finite element model and has to be explicitly defined; 2) probability density function $p_{\mathbf{Q}}(\mathbf{q})$ of random variable $\mathbf{Q}$ has to be constructed; 3) mappings $\mathbf{q} \mapsto[A(\mathbf{q}+\mathbf{q})]$, in which $[A]$ denotes $[\mathcal{M}],[\mathcal{D}]$ or $[\mathcal{K}]$, have to be explicitly constructed. These three steps constitute a parametric model of random uncertainties for the reduced matrix model. Concerning details related to such a parametric approach, we refer the reader to [8-22] for general developments, to [23-30] for applications, to [31-38] for general aspects related to stochastic finite elements. Some other aspects related to this kind of parametric models of random uncertainties can also be found in the context of developments written in stochastic dynamics and parametric stochastic excitations (see for instance [8,21,39-49]). Finally, it should be noted that there are some connections between the parametric model of random uncertainties in linear structural dynamics and stochastic linearization methods with random parameters for nonlinear dynamical systems [50-56].

\subsection{Introduction of a nonparametric model of random uncertainties in linear structural dynamics}

An interesting question concerns the possibility of directly constructing a random model of the generalized dynamic stiffness matrix of a structure or a substructure without having to determine the uncertain local parameters of the finite element model (for instance, the fuzzy structure theory $[6,57,58]$ belongs to this class of problem). In this paper, we propose an approach allowing a random uncertainties model to be constructed for a reduced matrix model in linear structural dynamics, introducing a probabilistic model for the generalized mass, damping and stiffness matrices obviating identification of uncertain local parameters $\mathbf{q}$ of the finite element model and therefore the construction of functions $\mathbf{q} \mapsto[A(\mathbf{q}+\mathbf{q})]$ in which $[A]$ denotes the generalized mass matrix $[\mathcal{M}]$, generalized damping matrix $[\mathcal{D}]$ or generalized stiffness matrix $[\mathcal{K}]$. This nonparametric model of random uncertainties is based on direct construction of a probability model of the generalized mass, damping and stiffness matrices, using only the available information which is constituted of the mean generalized mass, damping and stiffness matrices of the mean reduced matrix model. This paper describes the explicit construction of the theory of this nonparametric model. In Section 2, we introduce the principle of construction of a nonparametric model of random uncertainties in linear structural dynamics using the available information constituted of the mean reduced matrix model of the structure. Section 3 deals with the explicit construction of a probability model for symmetric positive-definite real random matrices using only the available information, and consequently, using the entropy optimization principle (which has been applied in another context for different problems [59-64]). This method allows the probabilistic model of the generalized mass, damping and stiffness matrices to be constructed. In Section 4, we finish the construction of the random generalized mass, damping and stiffness matrices and we present the complete development of the random reduced matrix model in the low-frequency range and an efficient representation of the probability model which is very well adapted to algebraic calculus and to Monte Carlo numerical simulation in order to compute the responses of the dynamical system with random uncertainties. In Section 5, we present a simple example. 
In this section, we introduce the principle of construction of a nonparametric model of random uncertainties, the available information being constituted of the mean reduced matrix model of the structure.

\subsection{Vector and matrix notations}

Any vector $\mathbf{x}=\left(x_{1}, \ldots, x_{n}\right)$ in Euclidean space $\mathbb{R}^{n}$ is identified with the $(n \times 1)$ column matrix of its $x_{j}$ components. Euclidean space $\mathbb{R}^{n}$ is equipped with the usual inner product $\left.<\mathbf{x}, \mathbf{y}\right\rangle=\mathbf{x}^{T} \mathbf{y}=\sum_{j=1}^{n} x_{j} y_{j}$ and the associated Euclidean norm $\|\mathbf{y}\|=<\mathbf{y}, \mathbf{y}>^{1 / 2}$, where $\mathbf{x}^{T}$ denotes the transpose of $\mathbf{x}$. Hermitian space $\mathbb{C}^{n}$ is equipped with the Hermitian inner product $(\mathbf{x}, \mathbf{y})=<\mathbf{x}, \overline{\mathbf{y}}>$ and the associated norm $\|\mathbf{y}\|=<\mathbf{y}, \overline{\mathbf{y}}\rangle^{1 / 2}$ where $\overline{\mathbf{y}}$ is the conjugate of $\mathbf{y}$ in $\mathbb{C}^{n}$. Let $\mathbb{K}$ be $\mathbb{R}$ or $\mathbb{C}$ and $\mathbb{M}_{n, m}(\mathbb{K})$ be the space of all the $(n \times m)$ matrices $[A]$ whose elements $A_{i j}=[A]_{i j}$ are in $\mathbb{K}$. If $n=m$, we denote $\mathbb{M}_{n}(\mathbb{K})$ as $\mathbb{M}_{n, n}(\mathbb{K})$. The determinant of matrix $[A]$ in $\mathbb{M}_{n}(\mathbb{K})$ is denoted as $\operatorname{det}[A]$ and its trace $\operatorname{tr}[A]=\sum_{j=1}^{n}[A]_{j j}$. The transpose of $[A]$ in $\mathbb{M}_{n}(\mathbb{K})$ is denoted as $[A]^{T}$ and its adjoint as $[A]^{*}=\overline{[A]}^{T}$. The Frobenius norm (or the Hilbert-Schmidt norm) of matrix $[A]$ in $\mathbb{M}_{n}(\mathbb{K})$ is defined by $\|[A]\|_{F}=\left(\operatorname{tr}\left\{[A][A]^{*}\right\}\right)^{1 / 2}$. Finally, we introduce the subspace $\mathbb{M}_{n}^{S}(\mathbb{K})$ of all the $(n \times n)$ symmetric matrices (a matrix $[A]$ in $\mathbb{M}_{n}^{S}(\mathbb{K})$ is such that $[A]=[A]^{T}$, i.e. $[A]_{i j}=[A]_{j i}$ ) and the subspace $\mathbb{M}_{n}^{+}(\mathbb{R})$ of $\mathbb{M}_{n}^{S}(\mathbb{R})$ constituted of all the positive-definite symmetric $(n \times n)$ real matrices.

\subsection{Nonparametric model of random uncertainties for the reduced matrix model matrices}

\subsubsection{Principle of construction}

The global construction of random uncertainties is associated with the mean finite element model defined in the frequency domain by Eq. (2) and consists in directly introducing mass, damping and stiffness random matrices $[\mathbf{M}],[\mathbf{D}]$ and $[\mathbf{K}]$. Consequently, in the frequency domain the finite element model with random uncertainties is written as

$$
\left(-\omega^{2}[\mathbf{M}]+i \omega[\mathbf{D}]+[\mathbf{K}]\right) \mathbf{Y}(\omega)=\mathbf{x}(\omega)
$$

in which $\mathbf{Y}(\omega)=\left(Y_{1}(\omega), \ldots, Y_{m}(\omega)\right)$ is the random vector with values in $\mathbb{C}^{m}$ constituted of the $m$ DOFs (displacements and/or rotations), $\mathbf{x}=\left(x_{1}, \ldots, x_{m}\right)$ is the vector of the $m$ inputs (forces and/or moments) defined in Section 1.1 and $[\mathbf{M}],[\mathbf{D}]$ and $[\mathbf{K}]$ are random matrices with values in $\mathbb{M}_{m}^{S}(\mathbb{R})$. It should be noted that the mean finite element model defined by Eq. (2) cannot be used to predict the reponse for all $\omega$ in $\mathbb{R}$ but only for $\omega$ in $\mathbb{B}$ for which the mean model has been developed. This means that the mean finite element model does not constitute an available information to construct the random finite element model for all $\omega$ in $\mathbb{R}$. On the other hand the mean reduced matrix model defined by Eqs. (6) and (7) constitutes the true available information to construct the response of the mean finite element model for $\omega$ in $\mathbb{B}$. Consequently, since the available information is constituted of the mean reduced matrix model, the probability model has not to be constructed for the random finite element model defined by Eq. (15) but for the random reduced matrix model associated with the mean reduced matrix model defined by Eqs. (6) and (7). This random reduced matrix model is then written as

$$
\left[\mathbf{H}_{N}(\omega)\right]=\sum_{\alpha=1}^{N} \sum_{\beta=1}^{N}[\mathcal{T}(\omega)]_{\alpha \beta} \mathbf{y}_{\alpha} \mathbf{y}_{\beta}^{T}
$$

in which $[\mathcal{T}(\omega)]$ is the $(N \times N)$ complex symmetric random matrix defined by

$$
[\mathcal{T}(\omega)]=\left(-\omega^{2}[\mathcal{M}]+i \omega[\mathcal{D}]+[\mathcal{K}]\right)^{-1},
$$

where $[\mathcal{M}],[\mathcal{D}]$ and $[\mathcal{K}]$ are the random generalized mass, damping and stiffness matrices which have to be symmetric positive-definite $(N \times N)$ real matrices, i.e. random matrices with values in $\mathbb{M}_{N}^{+}(\mathbb{R})$. Finally, we have to define the available information which is useful for constructing the probabilistic model.

\subsubsection{Available information}

Proba. Eng. Mech. 
The basic available information is the mean reduced matrix model which is constituted of the mean generalized mass, damping and stiffness matrices $[\underline{\mathcal{M}}],[\underline{\mathcal{D}}]$ and $[\underline{\mathcal{K}}]$ defined in Section 1.1 and which are in $M_{N}^{+}(\mathbb{R})$. Random generalized mass, damping and stiffness matrices $[\mathcal{M}],[\mathcal{D}]$ and $[\mathcal{K}]$ are second-order random variables with values in $M_{N}^{+}(\mathbb{R})$ such that

$$
E\{[\mathcal{M}]\}=[\underline{\mathcal{M}}] \quad, \quad E\{[\mathcal{D}]\}=[\underline{\mathcal{D}}] \quad, \quad E\{[\mathcal{K}]\}=[\underline{\mathcal{K}}] .
$$

In addition, we need to introduce information relative to the existence of moments of random variables $[\mathcal{M}]^{-1}$, $[\mathcal{D}]^{-1}$ and $[\mathcal{K}]^{-1}$ (such as second-order moments). Since random matrices $[\mathcal{M}],[\mathcal{D}]$ and $[\mathcal{K}]$ are almost surely positive definite, their inverses exist almost surely, but this property does not imply the existence of moments. We therefore introduce the following constraints,

$$
E\left\{\left\|[\mathcal{M}]^{-1}\right\|_{F}^{\gamma_{\mathcal{M}}}\right\}<+\infty \quad, \quad E\left\{\left\|[\mathcal{D}]^{-1}\right\|_{F}^{\gamma_{\mathcal{D}}}\right\}<+\infty \quad, \quad E\left\{\left\|[\mathcal{K}]^{-1}\right\|_{F}^{\gamma_{\mathcal{K}}}\right\}<+\infty \quad,
$$

in which $\gamma_{\mathcal{M}} \geq 1, \gamma_{\mathcal{D}} \geq 1$ and $\gamma_{\mathcal{K}} \geq 1$ are positive integers. We then have to construct a probability model for symmetric positive-definite real random matrices $[\mathcal{M}],[\mathcal{D}]$ and $[\mathcal{K}]$ with the available information defined by Eqs. (18) and (19). This construction is performed in Section 3 using the entropy optimization principle.

\section{Construction of a probability model for symmetric positive-definite real random matrices using the entropy optimization principle}

In this section, we construct a probability model of a random matrix with values in the $(n \times n)$ real symmetric positive-definite matrices.

\subsection{Probability density function on the space of positive-definite symmetric real matrices}

Let $[\mathbf{A}]$ be a random matrix with values in $\mathbb{M}_{n}^{+}(\mathbb{R}) \subset \mathbb{M}_{n}^{S}(\mathbb{R})$ whose probability distribution

$$
P_{[\mathbf{A}]}=p_{[\mathbf{A}]}([A]) \widetilde{d} A
$$

is defined by a probability density function $[A] \mapsto p_{[\mathbf{A}]}([A])$ from $\mathbb{M}_{n}^{+}(\mathbb{R})$ into $\mathbb{R}^{+}=[0,+\infty[$ with respect to the measure (volume element) $\widetilde{d} A$ on $\mathbb{M}_{n}^{S}(\mathbb{R})$ defined below. This probability density function is such that

$$
\int_{M_{n}^{+}(\mathbb{R})} p_{[\mathbf{A}]}([A]) \widetilde{d} A=1 .
$$

The objective of this section is to clearly define measure $\widetilde{d} A$ induced by the Euclidean structure on $\mathbb{M}_{n}^{S}(\mathbb{R})$.

\subsubsection{Euclidean structure and measure on space $\mathbb{R}^{n}$}

Space $\mathbb{R}^{n}$ is equipped with the Euclidean inner product defined in Section 2.1. Let $\left\{\mathbf{e}_{1}, \ldots, \mathbf{e}_{n}\right\}$ be the canonical basis of $\mathbb{R}^{n}$. Any vector $\mathbf{x}=\left(x_{1}, \ldots, x_{n}\right)$ in $\mathbb{R}^{n}$ can be written as $\mathbf{x}=\sum_{j=1}^{n} x_{j} \mathbf{e}_{j}$. Since $<\mathbf{e}_{i}, \mathbf{e}_{j}>=\delta_{i j}$ in which $\delta_{i j}=0$ if $i \neq j$ and $\delta_{j j}=1$, we deduce that $\left\{\mathbf{e}_{j}\right\}_{j}$ is an orthonormal basis in $\mathbb{R}^{n}$. This Euclidean structure on $\mathbb{R}^{n}$ defines the measure (volume element) $d \mathbf{x}$ on $\mathbb{R}^{n}$ such that $d \mathbf{x}=\prod_{j=1}^{n} d x_{j}$.

\subsubsection{Euclidean structure and measure on space $\mathbb{M}_{n}(\mathbb{R})$}

Any matrix $[A]$ in $\mathbb{M}_{n}(\mathbb{R})$ can be written as $[A]=\sum_{i, j=1}^{n} A_{i j}\left[b_{i j}\right]$ in which $A_{i j}=[A]_{i j}$ are the elements of matrix $[A]$ and $\left[b_{i j}\right]$ are matrices in $\mathbb{M}_{n}(\mathbb{R})$ such that $\left[b_{i j}\right]=\mathbf{e}_{i} \mathbf{e}_{j}^{T}$. If $[A]$ and $[B]$ are matrices in $\mathbb{M}_{n}(\mathbb{R})$, then their inner product can be defined by

$$
\ll[A],[B] \gg=\operatorname{tr}\left\{[A][B]^{T}\right\}=\sum_{i, j=1}^{n} A_{i j} B_{i j},
$$

and the associated norm is the Frobenius norm $\|[A]\|_{F}=\ll[A],[A] \gg^{1 / 2}$. Since $\ll\left[b_{i j}\right],\left[b_{i^{\prime} j^{\prime}}\right] \gg=\delta_{i i^{\prime}} \delta_{j j^{\prime}}$, we deduce that $\left\{\left[b_{i j}\right]\right\}_{i j}$ is an orthonormal basis in $M_{n}(\mathbb{R})$. This Euclidean structure on $\mathbb{M}_{n}(\mathbb{R})$ defines the measure (volume element) $d A$ on $\mathbb{M}_{n}(\mathbb{R})$ such that

$$
d A=\Pi_{i, j=1}^{n} d A_{i j}
$$


3.1.3. Euclidean structure and measure on space $\mathbb{M}_{n}^{S}(\mathbb{R})$

Space $M_{n}^{S}(\mathbb{R}) \subset \mathbb{M}_{n}(\mathbb{R})$ is constituted of the symmetric $(n \times n)$ real matrices. It can easily be verified that the family $\left\{\left[\widetilde{b}_{i j}\right], 1 \leq i \leq j \leq n\right\}$ of symmetric matrices defined by

$$
\left[\widetilde{b}_{i i}\right]=\mathbf{e}_{i} \mathbf{e}_{i}^{T} \quad, \quad\left[\widetilde{b}_{i j}\right]=\frac{1}{\sqrt{2}}\left(\mathbf{e}_{i} \mathbf{e}_{j}^{T}+\mathbf{e}_{j} \mathbf{e}_{i}^{T}\right) \quad \text { if } \quad i<j \quad,
$$

is an orthonormal basis in $\mathbb{M}_{n}^{S}(\mathbb{R})$ because $\ll\left[\widetilde{b}_{i j}\right],\left[\widetilde{b}_{i^{\prime} j^{\prime}}\right] \gg=\delta_{i i^{\prime}} \delta_{j j^{\prime}}$ for $i \leq j$ and $i^{\prime} \leq j^{\prime}$. Any symmetric matrix $[A]$ in $\mathbb{M}_{n}^{S}(\mathbb{R})$ can then be written as

$$
[A]=\sum_{1 \leq i \leq j \leq n} \widetilde{A}_{i j}\left[\widetilde{b}_{i j}\right]
$$

in which, for $1 \leq i \leq j \leq n$, real numbers $\widetilde{A}_{i j}$ are defined by

$$
\widetilde{A}_{i i}=A_{i i} \quad, \quad \widetilde{A}_{i j}=\sqrt{2} A_{i j} \quad \text { if } i<j \quad .
$$

If $[A]$ and $[B]$ are matrices in $\mathbb{M}_{n}^{S}(\mathbb{R}) \subset \mathbb{M}_{n}(\mathbb{R})$, then their inner product is written as

$$
\ll[A],[B] \gg=\operatorname{tr}\left\{[A][B]^{T}\right\}=\sum_{1 \leq i \leq j \leq n} \widetilde{A}_{i j} \widetilde{B}_{i j} .
$$

This Euclidean structure on $\mathbb{M}_{n}^{S}(\mathbb{R})$ defines the measure (volume element) $\widetilde{d} A$ on $\mathbb{M}_{n}^{S}(\mathbb{R})$ such that

$$
\widetilde{d} A=\Pi_{1 \leq i \leq j \leq n} d \widetilde{A}_{i j} .
$$

From Eqs. (25) and (27), we deduce that

$$
\widetilde{d} A=2^{n(n-1) / 4} \Pi_{1 \leq i \leq j \leq n} d A_{i j} .
$$

\subsection{Available information for construction of the probability model}

We are interested in the construction of the probability distribution of a second-order random variable $[\mathbf{A}]$ with values in $M_{n}^{+}(\mathbb{R})$ for which the available information is the mean value of random matrix $[\mathbf{A}]$,

$$
E\{[\mathbf{A}]\}=\int_{\mathbb{M}_{n}^{+}(\mathbb{R})}[A] p_{[\mathbf{A}]}([A]) \widetilde{d} A=[\underline{A}],
$$

in which $E$ denotes the mathematical expectation and where the mean value $[\underline{A}]$ is given in $\mathbb{M}_{n}^{+}(\mathbb{R})$. In addition, we assume that random matrix $[\mathbf{A}]$ is such that

$$
E\{\ln (\operatorname{det}[\mathbf{A}])\}=v \quad \text { with } \quad|v|<+\infty \quad .
$$

In Section 3.6, we prove that the constraint defined by Eq. (30) allows us to obtain the existence of moments related to the inverse random matrix $[\mathbf{A}]^{-1}$,

$$
E\left\{\left\|[\mathbf{A}]^{-1}\right\|_{F}^{\gamma}\right\}<+\infty,
$$

in which $\gamma \geq 1$ is a positive integer. Consequently, from Eqs. (21), (29) and (30), we deduce that the constraints imposed for the construction of the probability model of random matrix $[\mathbf{A}]$ with values in $\mathbb{M}_{n}^{+}(\mathbb{R})$ are

$$
\begin{gathered}
\int_{\mathbb{M}_{n}^{+}(\mathbb{R})} p_{[\mathbf{A}]}([A]) \widetilde{d} A=1, \\
\int_{\mathbb{M}_{n}^{+}(\mathbb{R})}[A] p_{[\mathbf{A}]}([A]) \widetilde{d} A=[\underline{A}] \in \mathbb{M}_{n}^{+}(\mathbb{R}), \\
\int_{\mathbb{M}_{n}^{+}(\mathbb{R})} \ln (\operatorname{det}[\mathbf{A}]) p_{[\mathbf{A}]}([A]) \widetilde{d} A=v \quad \text { with } \quad|v|<+\infty .
\end{gathered}
$$




\subsection{Introduction of the maximum entropy principle}

We use the maximum entropy principle to construct the probability model of random matrix $[\mathbf{A}]$ with values in $M_{n}^{+}(\mathbb{R})$ based only on the use of the available information defined in Section 3.2. In this section, we recall the maximum entropy principle. Let $Z$ be a discrete-valued random variable taking its values in $\mathcal{Z}=\left\{z_{i}\right\}_{i}$ with the corresponding probabilities $\left\{p_{i}\right\}_{i}$ in which $\mathbf{p}=\left\{p_{i}\right\}_{i}$ is the discrete probability distribution such that $p_{i} \geq 0$ and $\sum_{i} p_{i}=1$. Let $g_{1}, \ldots, g_{m}$ be functions from $\mathcal{Z}$ into $\mathbb{R}$ such that, for all $j$, the mathematical expectation $\underline{g}_{j}=E\left\{g_{j}(Z)\right\}=\sum_{i} p_{i} g_{j i}$ exists, in which $g_{j i}=g_{j}\left(z_{i}\right)$. The measure of entropy (uncertainty) $S(\mathbf{p})=-\sum_{i} p_{i} \ln p_{i} \geq 0$ of discrete probability distribution $\mathbf{p}$ was initially introduced by Shannon [65] in the context of the information theory. The maximum entropy principle for a discrete probability distribution was introduced by Jaynes [66]. This principle allows the probability distribution of a discrete-valued random variable to be explicitly constructed using only the available information, avoiding the use of any additional information which introduces a bias on the estimation of the probability distribution. This approach allows a coherent probability model to be constructed in the case where little objective information is available. Jayne's maximum entropy principle consists in maximizing the Shannon measure of entropy $S(\mathbf{p})=-\sum_{i} p_{i} \ln p_{i}$, subject to the $1+m$ constraint equations $\sum_{i} p_{i}-1=0$ and $\sum_{i} p_{i} g_{j i}-\underline{g}_{j}=0$ for $j$ in $\{1, \ldots, m\}$ in which $g_{j i}$ and $\underline{g}_{j}$ are given. It should be noted that the use of the Shannon measure of entropy implies that inequalities $p_{i} \geq 0$ are automatically satisfied. This optimization problem with constraints is solved by introducing $1+m$ Lagrange multipliers denoted as $\left(\mu_{0}-1\right), \mu_{1}, \ldots, \mu_{m}$ corresponding to the $1+m$ constraints and calculating the maximum of the Lagrangian $\mathcal{L}(\mathbf{p})$ defined by $\mathcal{L}(\mathbf{p})=S(\mathbf{p})-\left(\mu_{0}-1\right)\left\{\sum_{i} p_{i}-1\right\}-\sum_{j=1}^{m} \mu_{j}\left\{\sum_{i} p_{i} g_{j i}-\underline{g}_{j}\right\}$. Jayne's maximum entropy principle can be extended to the case of a probability density function $p(\mathbf{x})$ on $\mathbb{R}^{n}$ with respect to $d \mathbf{x}$ of an $\mathbb{R}^{n}$-valued random variable. In this case, the measure of "entropy" is defined by $S(p)=-\int_{\mathbb{R}^{n}} p(\mathbf{x}) \ln (p(\mathbf{x})) d \mathbf{x}$. Since $p(\mathbf{x})$ can be greater than $1, \ln p(\mathbf{x})$ can be positive, $S(p)$ can then be negative and consequently, $S(p)$ cannot represent an absolute measure of uncertainty (in general the measure of uncertainty must be positive). Nevertheless, it can be verified (see for instance [67]) that $S(p)$ measures a relative uncertainty.

\subsection{Probability model using the maximum entropy principle}

In this section, we construct probability density function $p_{[\mathbf{A}]}([A])$ and characteristic function $\Phi_{[\mathbf{A}]}([\Theta])$ of random matrix $[\mathbf{A}]$ with values in $\mathbb{M}_{n}^{+}(\mathbb{R})$, using the maximum entropy principle and the available information defined by Eqs. (32) to (34).

\subsubsection{Construction of the probability density function}

Let $\left(\mu_{0}-1\right),[\mu]$ and $(1-\lambda)$ be the Lagrange multipliers corresponding to the constraints defined by Eqs. (32), (33) and (34) respectively, with $\mu_{0} \in \mathbb{R},[\mu] \in \mathbb{M}_{n}^{+}(\mathbb{R})$ and $\lambda \in \mathbb{R}$. We then introduce the Lagrangian $\mathcal{L}\left(p_{[\mathbf{A}]}\right)=$ $S\left(p_{[\mathbf{A}]}\right)-\left(\mu_{0}-1\right) g_{0}-\sum_{i, j=1}^{n}[\mu]_{i j}[g]_{i j}-(1-\lambda) g_{1}$ in which $S\left(p_{[\mathbf{A}]}\right)=-\int_{\mathbb{M}_{n}^{+}(\mathbb{R})} p_{[\mathbf{A}]}([A]) \ln \left(p_{[\mathbf{A}]}([A])\right) \widetilde{d} A$ is the measure of entropy, $g_{0}$ and $g_{1}$ are the real constant numbers defined by $g_{0}=\int_{M_{n}^{+}(\mathbb{R})} p_{[\mathbf{A}]}([A]) \widetilde{d} A-1$ and $g_{1}=\int_{M_{n}^{+}(\mathbb{R})} \ln (\operatorname{det}[\mathbf{A}]) p_{[\mathbf{A}]}([A]) \widetilde{d} A-v$ and finally, $[g]$ is the $(n \times n)$ symmetric real matrix defined by $[g]=\int_{\mathbb{M}_{n}^{+}(\mathbb{R})}[A] p_{[\mathbf{A}]}([A]) \widetilde{d} A-[\underline{A}]$. The calculus of variations applied to Lagrangian $\mathcal{L}$ allows the maximum of $\mathcal{L}$ to be calculated and yields $p_{[\mathbf{A}]}([A])=\mathbb{1}_{\mathbb{M}_{n}^{+}}([A]) \times \exp \left(-\mu_{0}-\ll[\mu],[A] \gg-(1-\lambda) \ln (\operatorname{det}[A])\right)$ in which $\mathbb{1}_{\mathbb{M}_{n}^{+}(\mathbb{R})}([A])$ is equal to 1 if $[A] \in \mathbb{M}_{n}^{+}(\mathbb{R})$ and is equal to zero if $[A] \notin \mathbb{M}_{n}^{+}(\mathbb{R})$. Introducing the positive constant of normalization $c_{0}=\exp \left\{-\mu_{0}\right\}$, the probability density function can be rewritten as

$$
p_{[\mathbf{A}]}([A])=\mathbb{1}_{\mathbb{M}_{n}^{+}(\mathbb{R})}([A]) \times c_{0} \times(\operatorname{det}[A])^{\lambda-1} \times \exp (-\ll[\mu],[A] \gg) .
$$

Since the constraint defined by Eq. (34) has only been introduced to ensure that Eq. (31) is satisfied, there is no advantage of expressing $\lambda$ as a function of $v$. Consequently, $\lambda$ is considered as the new parameter instead of $v$, which has to be determined as a function of $n$ and $\gamma$ in order to satisfy Eq. (31). Constant $c_{0}$ of normalization and matrix $[\mu]$ have to be determined as a function of $\lambda$ and $[\underline{A}]$ using Eqs. (32) and (33). In order to perform this calculation, we need results concerning the Siegel integral for a positive-definite symmetric real matrix. 
We introduce the Siegel integral $J(\lambda,[\mu])$ defined for $\lambda>0$ and $[\mu]$ in $\mathbb{M}_{n}^{+}(\mathbb{R})$, corresponding to the multivariate generalization of the gamma function [68] and such that

$$
J(\lambda,[\mu])=\int_{\mathbb{M}_{n}^{+}(\mathbb{R})}(\operatorname{det}[A])^{\lambda-1} \exp (-\ll[\mu],[A] \gg) \widetilde{d} A
$$

For all real $\lambda>0$ and for all $[\mu]$ in $\mathbb{M}_{n}^{+}(\mathbb{R})$, we have $0<J(\lambda,[\mu])<+\infty$ and

$$
J(\lambda,[\mu])=(2 \pi)^{n(n-1) / 4} \frac{\left\{\Pi_{\ell=1}^{n} \Gamma\left(\frac{n-\ell+2 \lambda}{2}\right)\right\}}{(\operatorname{det}[\mu])^{(n-1+2 \lambda) / 2}},
$$

in which $\Gamma(\lambda)$ is the gamma function defined for $\Re e \lambda>0$ by

$$
\Gamma(\lambda)=\int_{0}^{+\infty} t^{\lambda-1} e^{-t} d t
$$

For $[\mu]=[I]$ and $n=1$, the Siegel integral coincides with the gamma function defined by Eq. (38). It should be noted that the Siegel integral is presently defined with respect to measure $\widetilde{d} A$ and not with respect to measure $\Pi_{1 \leq i \leq j \leq n} d A_{i j}$ (these two measures differ by a factor of $2^{n(n-1) / 4}$ ). A proof of Eq. (37) can be written in two steps. First, since $[\mu]$ belongs to $\mathbb{M}_{n}^{+}(\mathbb{R})$, there exists an orthogonal matrix $[\psi] \in \mathbb{M}_{n}(\mathbb{R})$ such that $[\psi]^{T}[\mu][\psi]=[\Lambda]$ in which $[\Lambda]$ is a diagonal matrix in $\mathbb{M}_{n}^{+}(\mathbb{R})$. Introducing the change of variable $[A]=[\psi][G][\psi]^{T}$ and since $\operatorname{det}[A]=\operatorname{det}[G]$ and $\widetilde{d} A=\widetilde{d} G$, Eq. (36) can be written as $J(\lambda,[\mu])=\int_{M_{n}^{+}(\mathbb{R})}(\operatorname{det}[G])^{\lambda-1} \exp (-\ll[\Lambda],[G] \gg$ ) $\widetilde{d} G$. Secondly, since $[G]$ belongs to $\mathbb{M}_{n}^{+}(\mathbb{R})$, there exists an upper triangular matrix $[L] \in \mathbb{M}_{n}(\mathbb{R})$ such that $[G]=[L]^{T}[L]$ with $[L]_{\ell \ell}>0$ for all $\ell$. For $\ell \leq \ell^{\prime}$, we define $\widetilde{L}_{\ell \ell^{\prime}}$ such that $\widetilde{L}_{\ell \ell}=L_{\ell \ell}$ and $\widetilde{L}_{\ell \ell^{\prime}}=\sqrt{2} L_{\ell \ell^{\prime}}$ if $\ell<\ell^{\prime}$. Let $\widetilde{d} L$ be the measure defined by $\widetilde{d} L=\Pi_{1 \leq \ell \leq \ell^{\prime} \leq n} d \widetilde{L}_{\ell \ell^{\prime}}=2^{n(n-1) / 4} \Pi_{1 \leq \ell \leq \ell^{\prime} \leq n} d L_{\ell \ell^{\prime}}$. Introducing the change of variable $[G]=[L]^{T}[L]$ in the integral over $\mathbb{M}_{n}^{+}(\mathbb{R})$ and since $\operatorname{det}[G]=\Pi_{\ell=1}^{n} \widetilde{L}_{\ell \ell}^{2}, \ll[\Lambda],[G] \gg=$ $\sum_{j=1}^{n} \Lambda_{j j}\left(\widetilde{L}_{j j}^{2}+\frac{1}{2} \sum_{\ell<j} \widetilde{L}_{\ell j}^{2}\right)$ and $\widetilde{d} G=2^{n}\left\{\Pi_{\ell=1}^{n} \widetilde{L}_{\ell \ell}^{n-\ell+1}\right\} \widetilde{d} L$ (for the proof, see Appendix A), we obtain Eq. $(37)$.

\subsubsection{Characteristic function}

For $\lambda>0$ and for all $[\Theta]$ in $M_{n}^{S}(\mathbb{R})$, the characteristic function of random matrix $[\mathbf{A}]$ with values in $M_{n}^{+}(\mathbb{R}) \subset M_{n}^{S}(\mathbb{R})$ is defined by $\Phi_{[\mathbf{A}]}([\Theta])=E\{\exp (i \ll[\Theta],[\mathbf{A}] \gg)\}$ and can then be written as

$$
\Phi_{[\mathbf{A}]}([\Theta])=\int_{M_{n}^{+}(\mathbb{R})} \exp (i \ll[\Theta],[A] \gg) p_{[\mathbf{A}]}([A]) \widetilde{d} A
$$

in which $p_{[\mathbf{A}]}([A])$ is given by Eq. (35) and is then written as

$$
\Phi_{[\mathbf{A}]}([\Theta])=\left\{\operatorname{det}\left(\left[I_{n}\right]-i[\mu]^{-1}[\Theta]\right)\right\}^{-(n-1+2 \lambda) / 2},
$$

where $\left[I_{n}\right]$ is the $(n \times n)$ unit matrix. The proof of Eq. (40) uses the Siegel integral. Since $[\mu]$ belongs to $\mathbb{M}_{n}^{+}(\mathbb{R})$, there exists $\varepsilon>0$ such that, for all $[V]$ in $\mathbb{M}_{n}^{S}(\mathbb{R})$ verifying $\|[V]\|_{F}<\varepsilon$, matrix $[\mu]-[V]$ is in $\mathbb{M}_{n}^{+}(\mathbb{R})$. Consequently, for $\lambda>0$, Siegel integral $J(\lambda,[\mu]-[V])$ is defined by Eq. (36). We can then consider the analytic extension of the mapping $[V] \mapsto J(\lambda,[\mu]-[V])$ to the space $M_{n}^{S}(\mathbb{C})$ by writing $[V]=\Re e[V]+i[\Theta]$ with $[\Theta] \in M_{n}^{S}(\mathbb{R})$ and $\|\Re e[V]\|_{F}<\varepsilon$. Taking $\Re e[V]=[0]$ in this extension and from Eqs. (35) and (39), we deduce that $\Phi_{[\mathbf{A}]}([\Theta])=c_{0} J(\lambda,[\mu]-i[\Theta])$. From the equation $\Phi_{[\mathbf{A}]}([0])=1$, we can deduce the value of constant $c_{0}$ which is written as

$$
c_{0}=\frac{1}{J(\lambda,[\mu])}=(2 \pi)^{-n(n-1) / 4} \frac{(\operatorname{det}[\mu])^{(n-1+2 \lambda) / 2}}{\left\{\Pi_{\ell=1}^{n} \Gamma\left(\frac{n-\ell+2 \lambda}{2}\right)\right\}},
$$

Proba. Eng. Mech. 
and consequently, $\Phi_{[\mathbf{A}]}([\Theta])=J(\lambda,[\mu]-i[\Theta]) \times J(\lambda,[\mu])^{-1}$. From Eq. (37), we then deduce Eq. (40).

3.4.4. Second-order moments of random matrix $[\mathbf{A}]$

For all $[\Theta]$ in $\mathbb{M}_{n}^{S}(\mathbb{R}), j \leq k$ and $j^{\prime} \leq k^{\prime}$, we have

$$
\begin{gathered}
E\left\{\mathbf{A}_{j k}\right\}=\frac{-i}{\left(2-\delta_{j k}\right)}\left\{\frac{\partial}{\partial \Theta_{j k}} \Phi_{[\mathbf{A}]}([\Theta])\right\}_{[\Theta]=[0]}, \\
E\left\{\mathbf{A}_{j k} \mathbf{A}_{j^{\prime} k^{\prime}}\right\}=\frac{-1}{\left(2-\delta_{j k}\right)\left(2-\delta_{j^{\prime} k^{\prime}}\right)}\left\{\frac{\partial^{2}}{\partial \Theta_{j k} \partial \Theta_{j^{\prime} k^{\prime}}} \Phi_{[\mathbf{A}]}([\Theta])\right\}_{[\Theta]=[0]} .
\end{gathered}
$$

The calculation of the right-hand side of Eqs. (42) and (43) requires calculating the derivatives of a determinant. If $[H] \in M_{n}(\mathbb{C})$ and if $\|[H]\|_{F} \rightarrow 0$, then (see for instance [69]), $\operatorname{det}([I]+[H])=1+\operatorname{tr}[H]+\mathcal{O}\left(\|[H]\|_{F}^{2}\right.$ ). Using differential calculus, it can easily be shown that, for all invertible matrices $[B]$ in $M_{n}(\mathbb{C}), \partial(\operatorname{det}[B]) / \partial B_{j k}=$ $(\operatorname{det}[B])\left\{[B]^{-1}\right\}_{k j}$. From Eqs. (40), (42) and (43) and for $\lambda>0$, we deduce that

$$
\begin{gathered}
E\left\{\mathbf{A}_{j k}\right\}=\frac{(n-1+2 \lambda)}{2}\left\{[\mu]^{-1}\right\}_{j k}, \\
E\left\{\mathbf{A}_{j k} \mathbf{A}_{j^{\prime} k^{\prime}}\right\}=E\left\{\mathbf{A}_{j k}\right\} E\left\{\mathbf{A}_{j^{\prime} k^{\prime}}\right\} \\
+\frac{1}{(n-1+2 \lambda)}\left\{E\left\{\mathbf{A}_{j^{\prime} k}\right\} E\left\{\mathbf{A}_{j k^{\prime}}\right\}+E\left\{\mathbf{A}_{j j^{\prime}}\right\} E\left\{\mathbf{A}_{k k^{\prime}}\right\}\right\} .
\end{gathered}
$$

\subsubsection{Calculation of matrix parameter $[\mu]$}

Using Eq. (33) and Eq. (44), parameter $[\mu]$ can be expressed in terms of mean value $[\underline{A}] \in \mathbb{M}_{n}^{+}(\mathbb{R})$ of random matrix $[\mathbf{A}]$ with values in $\mathbb{M}_{n}^{+}(\mathbb{R})$,

$$
[\mu]=\frac{(n-1+2 \lambda)}{2}[\underline{A}]^{-1} \quad, \quad \lambda>0
$$

3.4.6. Characteristic function and probability density function of positive-definite random matrix $[\mathbf{A}]$ For $\lambda>0$ and $[\Theta] \in M_{n}^{S}(\mathbb{R})$, Eqs. (40) and (46) yield

$$
\Phi_{[\mathbf{A}]}([\Theta])=\left\{\operatorname{det}\left(\left[I_{n}\right]-\frac{2 i}{(n-1+2 \lambda)}[\underline{A}][\Theta]\right)\right\}^{-(n-1+2 \lambda) / 2}
$$

and substituting Eqs. (41) and (46) into Eq. (35) yields

$$
p_{[\mathbf{A}]}([A])=\mathbb{1}_{\mathfrak{M}_{n}^{+}(\mathbb{R})}([A]) \times c_{A} \times(\operatorname{det}[A])^{\lambda-1} \times \exp \left(-\frac{(n-1+2 \lambda)}{2} \ll[\underline{A}]^{-1},[A] \gg\right)
$$

in which positive constant $c_{A}$ is written as

$$
c_{A}=\frac{(2 \pi)^{-n(n-1) / 4}\left(\frac{n-1+2 \lambda}{2}\right)^{n(n-1+2 \lambda) / 2}}{\left\{\Pi_{\ell=1}^{n} \Gamma\left(\frac{n-\ell+2 \lambda}{2}\right)\right\}(\operatorname{det}[\underline{A}])^{(n-1+2 \lambda) / 2}} .
$$


3.4.7. Covariance tensor of random matrix $[\mathbf{A}]$

Since $E\{[\mathbf{A}]\}=[\underline{A}]$ and using Eq. (45), the covariance $C_{j k, j^{\prime} k^{\prime}}=E\left\{\left(\mathbf{A}_{j k}-\underline{A}_{j k}\right)\left(\mathbf{A}_{j^{\prime} k^{\prime}}-\underline{A}_{j^{\prime} k^{\prime}}\right)\right\}$ of random variables $\mathbf{A}_{j k}$ and $\mathbf{A}_{j^{\prime} k^{\prime}}$ is written as

$$
C_{j k, j^{\prime} k^{\prime}}=\frac{1}{(n-1+2 \lambda)}\left\{\underline{A}_{j^{\prime} k} \underline{A}_{j k^{\prime}}+\underline{A}_{j j^{\prime}} \underline{A}_{k k^{\prime}}\right\},
$$

and the variance $\sigma_{j k}^{2}=C_{j k, j k}$ of random variable $\mathbf{A}_{j k}$ is such that

$$
\sigma_{j k}^{2}=\frac{1}{(n-1+2 \lambda)}\left\{\underline{A}_{j k}^{2}+\underline{A}_{j j} \underline{A}_{k k}\right\} .
$$

Let $\delta_{A}$ be defined by

$$
\delta_{A}=\left\{\frac{E\left\{\|[\mathbf{A}]-[\underline{A}]\|_{F}^{2}\right\}}{\|[\underline{A}]\|_{F}^{2}}\right\}^{1 / 2} .
$$

Since $E\left\{\|[\mathbf{A}]-[\underline{A}]\|_{F}^{2}\right\}=\sum_{j} \sum_{k} \sigma_{j k}^{2}$, we deduce that

$$
\delta_{A}=\left\{\frac{1}{n-1+2 \lambda}\left(1+\frac{(\operatorname{tr}[\underline{A}])^{2}}{\operatorname{tr}\left([\underline{A}]^{2}\right)}\right)\right\}^{1 / 2} .
$$

\subsubsection{Remark concerning parameter $\lambda$}

For $n$ fixed, if $\lambda \rightarrow+\infty$, then $\delta_{A}$ and $\sigma_{j k} \rightarrow 0$ and therefore $[\mathbf{A}] \rightarrow[\underline{A}]$ almost surely. Consequently, parameter $\lambda$ allows the dispersion of the probability model to be controlled. If $[\underline{A}]=\left[I_{n}\right]$, then Eq. (51) yields $\delta_{A}=\{(1+1 / n) /(1-1 / n+2 \lambda / n)\}^{1 / 2}$. Consequently, if $n \gg 1$, we then have $\delta_{A} \sim(1+2 \lambda / n)^{-1 / 2}$ which shows that $\lambda \rightarrow+\infty$ means $\lambda / n \gg 1$. Concerning the calculation of the range of parameter $\lambda$ required to satisfy Eq. (31), we need a result concerning the probability distribution of eigenvalues of matrix $[\mathbf{A}]$ that we present in the next section.

\subsection{Probability distribution of the eigenvalues of random matrix $[\mathbf{A}]$ with values in $M_{n}^{+}(\mathbb{R})$}

Since mean value $[\underline{A}]=E\{[\mathbf{A}]\}$ is a matrix belonging to $\mathbb{M}_{n}^{+}(\mathbb{R})$, there exists an orthogonal matrix $[\underline{F}]$ verifying $\operatorname{det}[\underline{F}]=1$ and $[\underline{F}][\underline{F}]^{T}=[\underline{F}]^{T}[\underline{F}]=\left[I_{n}\right]$, such that $[\underline{A}]=[\underline{F}][\underline{B}][\underline{F}]^{T}$ in which $[\underline{B}]$ is a diagonal matrix whose diagonal is constituted of the positive eigenvalues $\underline{b}_{1}, \ldots, \underline{b}_{n}$ of matrix $[\underline{A}]$. Let us introduce the random matrix $[\mathbf{B}]$ with values in $M_{n}^{+}(\mathbb{R})$ such that $[\mathbf{A}]=[\underline{F}][\mathbf{B}][\underline{F}]^{T}$. We deduce that mean value $E\{[\mathbf{B}]\}$ of random matrix $[\mathbf{B}]$ is $[\underline{B}]$. It should be noted that random matrix $[\mathbf{B}]$ is not a diagonal matrix. From Eqs. (48) and (49), we deduce that the probability distribution of random matrix $[\mathbf{B}]$ is written as $P_{[\mathbf{B}]}=p_{[\mathbf{B}]}([B]) \widetilde{d} B$ in which probability density function $p_{[\mathbf{B}]}([B])$ is such that

$$
p_{[\mathbf{B}]}([B])=\mathbb{1}_{\mathbb{M}_{n}^{+}(\mathbb{R})}([B]) \times c_{B} \times(\operatorname{det}[B])^{\lambda-1} \times \exp \left(-\frac{(n-1+2 \lambda)}{2} \ll[\underline{B}]^{-1},[B] \gg\right),
$$

in which positive constant $c_{B}$ is written as

$$
c_{B}=\frac{(2 \pi)^{-n(n-1) / 4}\left(\frac{n-1+2 \lambda}{2}\right)^{n(n-1+2 \lambda) / 2}}{\left\{\Pi_{\ell=1}^{n} \Gamma\left(\frac{n-\ell+2 \lambda}{2}\right)\right\}(\operatorname{det}[\underline{B}])^{(n-1+2 \lambda) / 2}} .
$$

Since $[\mathbf{B}]$ is a random matrix with values in $\mathbf{M}_{n}^{+}$, there exists a random matrix $[\mathbf{S}]$ verifying $\operatorname{det}[\mathbf{S}]=1$ and $[\mathbf{S}][\mathbf{S}]^{T}=[\mathbf{S}]^{T}[\mathbf{S}]=\left[I_{n}\right]$ almost surely, such that $[\mathbf{B}]=[\mathbf{S}][\mathbf{\Sigma}][\mathbf{S}]^{T}$ in which $[\boldsymbol{\Sigma}]$ is a random diagonal matrix whose diagonal is constituted of the positive-valued random eigenvalues $\boldsymbol{\Sigma}_{1}, \ldots, \boldsymbol{\Sigma}_{n}$ of random matrix $[\mathbf{B}]$ (it should be noted that the mean value of random matrix $[\mathbf{B}]$ is not equal to the mean value of the diagonal random matrix $[\boldsymbol{\Sigma}]$ ). We deduce that

$$
[\mathbf{A}]=[\mathbf{R}][\mathbf{\Sigma}][\mathbf{R}]^{T},
$$

in which $[\mathbf{R}]$ is a random matrix defined by $[\mathbf{R}]=[\underline{F}][\mathbf{S}]$ such that $[\mathbf{R}][\mathbf{R}]^{T}=[\mathbf{R}]^{T}[\mathbf{R}]=\left[I_{n}\right]$ almost surely. From Eq. (54), we then deduce that diagonal random matrix $[\boldsymbol{\Sigma}]$ is constituted of the random eigenvalues of random matrix $[\mathbf{A}]$. In order to construct the probability distribution of the random eigenvalues $\boldsymbol{\Sigma}_{1}, \ldots, \boldsymbol{\Sigma}_{n}$ of random matrix $[\mathbf{A}]$, we need additional results concerning representation of orthogonal matrices. 
Let $[S]$ be any orthogonal matrix in $M_{n}(\mathbb{R})$ such that

$$
\operatorname{det}[S]=1 \quad, \quad[S][S]^{T}=[S]^{T}[S]=\left[I_{n}\right] .
$$

It is known that orthogonal matrix $[S]$ can be written as $[S]=\exp (\rho[T])$ in which $\rho \geq 0$ and where $[T]$ is a skew-symmetric real matrix in $M_{n}(\mathbb{R})$ having a Frobenius norm equal to 1 ,

$$
[T]^{T}=-[T] \quad, \quad\|[T]\|_{F}=1 .
$$

Due to Eq. (56), matrix $[T]$ depends only on $\nu=n(n-1) / 2-1$ independent parameters denoted as $\mathbf{r}=$ $\left(r_{1}, \ldots, r_{\nu}\right)$ and is then rewritten as $[T(\mathbf{r})]$. Consequently, matrix $[S]$ which depends only on $1+\nu=n(n-1) / 2$ independent parameters $\rho$ and $\mathbf{r}$, is then rewritten as

$$
[S(\rho, \mathbf{r})]=\exp (\rho[T(\mathbf{r})]) .
$$

It can be shown that there exist a choice of vector-valued parameter $\mathbf{r}$, a bounded subset $\mathcal{D}_{\rho} \subset \mathbb{R}^{+}$and a bounded subset $\mathcal{D}_{\mathbf{r}} \subset \mathbb{R}^{\nu}$ such that the range of the mapping $(\rho, \mathbf{r}) \mapsto[S(\rho, \mathbf{r})]$ defined on $\mathcal{D}_{\rho} \times \mathcal{D}_{\mathbf{r}}$, coincides with the set of all the orthogonal matrices in $M_{n}(\mathbb{R})$ verifying Eq. (55).

\subsubsection{Infinitesimal orthogonal matrices}

For $\mathbf{r}$ fixed in $\mathcal{D}_{\mathbf{r}}$ and from Eq. (57), we deduce that $[S(\rho, \mathbf{r})] \rightarrow\left[I_{n}\right]$ if $\rho \rightarrow 0$, and for $0<\rho \ll 1$, we can write

$$
[S(\rho, \mathbf{r})]=\left[I_{n}\right]+\rho[T(\mathbf{r})]+\mathcal{O}\left(\rho^{2}\right) .
$$

Let $[\Sigma]$ be any matrix in $\mathbb{M}_{n}(\mathbb{R})$ and let $[S(\rho, \mathbf{r})]$ be the infinitesimal orthogonal matrix defined by Eq. (58). Therefore, it can easily be verified that $[S(\rho, \mathbf{r})][\Sigma][S(\rho, \mathbf{r})]^{T}=[\Sigma]+\rho([T(\mathbf{r})][\Sigma]-[\Sigma][T(\mathbf{r})])+\mathcal{O}\left(\rho^{2}\right)$. If $[\Sigma]$ is a diagonal matrix whose diagonal elements are denoted as $\sigma_{1}, \ldots, \sigma_{n}$, then

$$
\sum_{k=1}^{n} \sigma_{k}[S(\rho, \mathbf{r})]_{j k}^{2}=\sigma_{j}+\mathcal{O}\left(\rho^{2}\right) \quad, \quad \forall j
$$

\subsubsection{Probability density function of the random eigenvalues}

Any matrix $[B]$ belonging to $M_{n}^{+}(\mathbb{R})$ can be written as

$$
[B]=[S(\rho, \mathbf{r})][\Sigma(\boldsymbol{\sigma})][S(\rho, \mathbf{r})]^{T},
$$

in which $[S(\rho, \mathbf{r})] \in \mathbb{M}_{n}(\mathbb{R})$ is defined by Eq. (57) and where $[\Sigma(\boldsymbol{\sigma})]$ is a diagonal matrix in $\mathbb{M}_{n}^{+}(\mathbb{R})$ constituted of all the positive eigenvalues $\sigma_{1}, \ldots, \sigma_{n}$ of matrix $[B]$ such that $[\Sigma(\boldsymbol{\sigma})]_{j k}=\sigma_{j} \delta_{j k}$ in which $\boldsymbol{\sigma}=\left(\sigma_{1}, \ldots, \sigma_{n}\right) \in$ $\mathcal{D}_{\boldsymbol{\sigma}}=(] 0,+\infty[)^{n} \subset \mathbb{R}^{n}$. Using Eq. (25), we associate matrix $[\widetilde{B}]$ with matrix $[B]$ defined by Eq. (60). Let $\boldsymbol{\beta}$ be the vector in $\mathbb{R}^{n(n+1) / 2}$ such that $\boldsymbol{\beta}=\left(B_{j k}, 1 \leq j \leq k \leq n\right)$. Let $\widetilde{d} B$ be the measure on $\mathbb{M}_{n}^{S}(\mathbb{R})$ defined by Eq. (28) such that $\widetilde{d} B=2^{n(n-1) / 4} d \boldsymbol{\beta}$ in which $d \boldsymbol{\beta}=\Pi_{1 \leq i \leq j \leq n} d B_{i j}$. Let $(\boldsymbol{\sigma}, \rho, \mathbf{r}) \mapsto \mathbf{f}(\boldsymbol{\sigma}, \rho, \mathbf{r})$ be the mapping from $\mathcal{D}_{\boldsymbol{\sigma}} \times \mathcal{D}_{\rho} \times \mathcal{D}_{\mathbf{r}}$ into $\mathbb{R}^{n(n+1) / 2}$ such that $\boldsymbol{\beta}=\mathbf{f}(\boldsymbol{\sigma}, \rho, \mathbf{r})$. The Jacobian matrix of mapping $\mathbf{f}$, denoted as $[J(\boldsymbol{\sigma}, \rho, \mathbf{r})]$, is such that for $1 \leq j \leq k \leq n$ and $1 \leq \ell \leq n(n+1) / 2$ we have $[J(\boldsymbol{\sigma}, \rho, \mathbf{r})]_{j k, \ell}=\partial B_{j k} / \partial x_{\ell}$ in which $\mathbf{x}=\left(x_{1}, \ldots, x_{n(n+1) / 2}\right)$ is defined by $\mathbf{x}=(\boldsymbol{\sigma}, \rho, \mathbf{r})$. Consequently, we have $d \boldsymbol{\beta}=|\operatorname{det}[J(\boldsymbol{\sigma}, \rho, \mathbf{r})]| d \boldsymbol{\sigma} d \rho d \mathbf{r}$. It can be proved [70,72] that $|\operatorname{det}[J(\boldsymbol{\sigma}, \rho, \mathbf{r})]|=g(\rho, \mathbf{r})\left\{\Pi_{j<k}\left|\sigma_{k}-\sigma_{j}\right|\right\}$ in which $g(\rho, \mathbf{r})$ does not depend on $\boldsymbol{\sigma}$ and is a bounded function on bounded sets $\mathcal{D}_{\rho} \times \mathcal{D}_{\mathbf{r}}$,

$$
0 \leq g(\rho, \mathbf{r}) \leq c_{g}<+\infty \quad, \quad \forall \rho \in \mathcal{D}_{\rho}, \forall \mathbf{r} \in \mathcal{D}_{\mathbf{r}}
$$

We then deduce that

$$
\widetilde{d} B=2^{n(n-1) / 4} g(\rho, \mathbf{r})\left\{\Pi_{j<k}\left|\sigma_{k}-\sigma_{j}\right|\right\} d \boldsymbol{\sigma} d \rho d \mathbf{r} .
$$

Proba. Eng. Mech. 
Using Eqs. (60) and (62) yields

$$
\begin{aligned}
p_{[\mathbf{B}]}([B]) \widetilde{d} B= & p_{[\mathbf{B}]}\left([S(\rho, \mathbf{r})][\Sigma(\boldsymbol{\sigma})][S(\rho, \mathbf{r})]^{T}\right) \\
& \times 2^{n(n-1) / 4} g(\rho, \mathbf{r})\left\{\Pi_{j<k}\left|\sigma_{k}-\sigma_{j}\right|\right\} d \boldsymbol{\sigma} d \rho d \mathbf{r},
\end{aligned}
$$

in which $p_{[\mathbf{B}]}([B])$ is defined by Eqs. (52) and (53). The probability density function $p_{[\boldsymbol{\Sigma}]}(\boldsymbol{\sigma})$ on $\left.\mathcal{D}_{\boldsymbol{\sigma}}=\right] 0,+\infty\left[{ }^{n} \subset\right.$ $\mathbb{R}^{n}$ with respect to Lebesgue measure $d \boldsymbol{\sigma}=d \sigma_{1} \ldots d \sigma_{n}$ of random diagonal matrix $[\boldsymbol{\Sigma}]$ (whose diagonal is constituted of the random eigenvalues of random matrix $[\mathbf{A}])$ is then written as $p_{[\boldsymbol{\Sigma}]}(\boldsymbol{\sigma})=\mathbb{1}_{\mathcal{D}_{\boldsymbol{\sigma}}}(\boldsymbol{\sigma}) 2^{n(n-1) / 4}$ $\times \int_{\mathcal{D}_{\rho}} \int_{\mathcal{D}_{\mathbf{r}}} p_{[\mathbf{B}]}\left([S(\rho, \mathbf{r})][\Sigma(\boldsymbol{\sigma})][S(\rho, \mathbf{r})]^{T}\right) \times g(\rho, \mathbf{r})\left\{\Pi_{j<k}\left|\sigma_{k}-\sigma_{j}\right|\right\} d \rho d \mathbf{r}$. For $\lambda>0$, substituting Eq. (52) yields

$$
\begin{aligned}
p_{[\Sigma]}(\boldsymbol{\sigma}) & =\mathbb{1}_{\mathcal{D}_{\boldsymbol{\sigma}}}(\boldsymbol{\sigma}) \times 2^{n(n-1) / 4} \times c_{B} \times \int_{\mathcal{D}_{\rho}} \int_{\mathcal{D}_{\mathbf{r}}} g(\rho, \mathbf{r}) \times\left(\sigma_{1} \times \ldots \times \sigma_{n}\right)^{\lambda-1} \\
& \times\left\{\Pi_{j<k}\left|\sigma_{k}-\sigma_{j}\right|\right\} \times \exp \left(-\frac{(n-1+2 \lambda)}{2} \sum_{j, k=1}^{n} \frac{\sigma_{k}}{\underline{b}_{j}}[S(\rho, \mathbf{r})]_{j k}^{2}\right) d \rho d \mathbf{r},
\end{aligned}
$$

in which $c_{B}$ is defined by Eq. (53). It should be noted that $\Pi_{j<k}\left(\sigma_{k}-\sigma_{j}\right)$ can be expressed with the Vandermonde determinant formula,

$$
\Pi_{j<k}\left(\sigma_{k}-\sigma_{j}\right)=\operatorname{det}\left[\begin{array}{cccc}
1 & \cdot & \cdot & 1 \\
\sigma_{1} & \cdot & \cdot & \sigma_{n} \\
\sigma_{1}^{2} & \cdot & \cdot & \sigma_{n}^{2} \\
\cdot & \cdot & \cdot & \cdot \\
\sigma_{1}^{n-1} & \cdot & \cdot & \sigma_{n}^{n-1}
\end{array}\right] .
$$

\subsubsection{Particular cases}

1) As a first particular case, we assume that mean matrix $[\underline{A}]$ has one eigenvalue of multiplicity $n$, i.e. we have $\underline{b}_{1}=\ldots=\underline{b}_{n}=\underline{b}$. Since $\sum_{j=1}^{n}[S(\rho, \mathbf{r})]_{j k}^{2}=1$ for all $k$ and taking into account Eq. (61), we deduce that

$$
\begin{aligned}
p_{[\Sigma]}(\boldsymbol{\sigma}) & =\mathbb{1}_{\mathcal{D}_{\boldsymbol{\sigma}}}(\boldsymbol{\sigma}) \times c_{\Sigma} \times\left(\sigma_{1} \times \ldots \times \sigma_{n}\right)^{\lambda-1} \\
& \times\left\{\Pi_{j<k}\left|\sigma_{k}-\sigma_{j}\right|\right\} \times \exp \left(-\frac{(n-1+2 \lambda)}{2 \underline{b}} \sum_{j=1}^{n} \sigma_{j}\right),
\end{aligned}
$$

in which $c_{\Sigma}$ is the constant of normalization such that

$$
\int_{\mathcal{D}_{\sigma}} p_{[\Sigma]}(\boldsymbol{\sigma}) d \boldsymbol{\sigma}=1
$$

The very interesting Eq. (66) shows the repulsion phenomenon of the eigenvalues due to random uncertainties. Figure 1 shows the contour plot composed of lines of constant values for the function $\left(\sigma_{1}, \sigma_{2}\right) \mapsto c_{\Sigma}^{-1} p_{[\Sigma]}\left(\sigma_{1}, \sigma_{2}\right)$ with $n=2$ and $\underline{b}=5 / 2$ (it should be noted that $p_{[\Sigma]}\left(\sigma_{1}, \sigma_{2}\right)=0$ for $\left.\sigma_{1}=\sigma_{2}\right)$.

2) The second particular case corresponds to large values of $\lambda$ which means that the dispersion of random matrix $[\mathbf{A}]$ is small (see Section 3.4.8). Since $[\underline{A}]=[\underline{F}][\underline{B}][\underline{F}]^{T}$ and $[\mathbf{A}]=[\underline{F}][\mathbf{B}][\underline{F}]^{T}$, and since $[\mathbf{A}] \rightarrow[\underline{A}]$ almost surely when $\lambda \rightarrow+\infty$, we then deduce that $[\mathbf{B}] \rightarrow[\underline{B}]$ almost surely when $\lambda \rightarrow+\infty$ (it is recalled that $[\underline{B}]$ is a diagonal matrix constituted of eigenvalues $\underline{b}_{1}, \ldots, \underline{b}_{n}$ of matrix $[\underline{A}]$ ). Since $[\mathbf{B}]=[\mathbf{S}][\mathbf{\Sigma}][\mathbf{S}]^{T}$, we deduce that $[\mathbf{S}] \rightarrow\left[I_{n}\right]$ almost surely when $\lambda \rightarrow+\infty$ and consequently, for large values of $\lambda$, random matrix $[\mathbf{S}]=[S(\rho, \mathbf{r})]=\exp (\rho[T(\mathbf{r})]))$ is an infinitesimal othogonal random matrix (this means that subset $\mathcal{D}_{\rho}$ can be written as $\mathcal{D}_{\rho}=\left[0, \rho_{0}\right]$ with $0<\rho_{0} \ll 1$ ). Consequently, we can substitute Eq. (59) into Eq. (64) and taking into account Eq. (61), we obtain the following approximation of the probability density function of the eigenvalues $\boldsymbol{\Sigma}_{1}, \ldots, \boldsymbol{\Sigma}_{n}$ of random matrix $[\mathbf{A}]=[\mathbf{R}][\mathbf{\Sigma}][\mathbf{R}]^{T}$ with $[\mathbf{R}]=[\underline{F}][\mathbf{S}]$,

$$
\begin{aligned}
p_{[\boldsymbol{\Sigma}]}(\boldsymbol{\sigma}) & =\mathbb{1}_{\mathcal{D}_{\boldsymbol{\sigma}}}(\boldsymbol{\sigma}) \times c_{\Sigma} \times\left(\sigma_{1} \times \ldots \times \sigma_{n}\right)^{\lambda-1} \\
& \times\left\{\Pi_{j<k}\left|\sigma_{k}-\sigma_{j}\right|\right\} \times \exp \left(-\frac{(n-1+2 \lambda)}{2} \sum_{j=1}^{n} \frac{\sigma_{j}}{\underline{b}_{j}}\right),
\end{aligned}
$$

in which $c_{\Sigma}$ is another constant of normalization defined by Eq. (67). 


\subsection{Range of parameter $\lambda$}

We have to determine the range of parameter $\lambda$ required to satisfy Eq. (31), in which $\gamma \geq 1$ is a positive integer. Since $[\mathbf{A}]=[\mathbf{R}][\mathbf{\Sigma}][\mathbf{R}]^{T}$ in which $[\mathbf{R}]$ is an orthogonal random matrix, we have $\|[\mathbf{A}]\|_{F}^{2}=\boldsymbol{\Sigma}_{1}^{2}+\ldots+\boldsymbol{\Sigma}_{n}^{2}$ and $\left\|[\mathbf{A}]^{-1}\right\|_{F}^{2}=\left(\boldsymbol{\Sigma}_{1}^{2}+\ldots+\boldsymbol{\Sigma}_{n}^{2}\right)^{-1}$ and consequently,

$$
E\left\{\left\|[\mathbf{A}]^{-1}\right\|_{F}^{\gamma}\right\}=\int_{\mathcal{D}_{\sigma}} \frac{p_{[\Sigma]}(\boldsymbol{\sigma}) d \boldsymbol{\sigma}}{\left(\sigma_{1}^{2}+\ldots+\sigma_{n}^{2}\right)^{\gamma / 2}}
$$

in which $\left.\mathcal{D}_{\boldsymbol{\sigma}}=\right] 0,+\infty\left[{ }^{n}\right.$. Since $0 \leq g(\rho, \mathbf{r}) \leq c_{g}<+\infty$ on bounded set $\mathcal{D}_{\rho} \times \mathcal{D}_{\mathbf{r}}$ and since $((n-1+$ $2 \lambda) / 2) \sum_{j, k=1}^{n}\left(\sigma_{k} / \underline{b}_{j}\right)[S(\rho, \mathbf{r})]_{j k}^{2} \geq 0$ because $\lambda>0$, from Eqs. (69) and (64), we deduce that $E\left\{\left\|[\mathbf{A}]^{-1}\right\|_{F}^{\gamma}\right\}<$ $+\infty$ if and only if

$$
\mathcal{I}_{\varepsilon}=\int_{\|\boldsymbol{\sigma}\|<\varepsilon} \frac{\left(\sigma_{1} \times \ldots \times \sigma_{n}\right)^{\lambda-1}}{\left(\sigma_{1}^{2}+\ldots+\sigma_{n}^{2}\right)^{\gamma / 2}} \times\left\{\Pi_{j<k}\left|\sigma_{k}-\sigma_{j}\right|\right\} d \boldsymbol{\sigma}<+\infty
$$

in which $0<\varepsilon \ll 1$. Introducing polar coordinates $r$ and $\boldsymbol{\theta}=\left(\theta_{1}, \ldots, \theta_{n-1}\right)$, we have (see Appendix B) $d \boldsymbol{\sigma}=r^{n-1} h_{1}(\boldsymbol{\theta}) d r d \boldsymbol{\theta}$. From Eq. (65), we deduce that $\Pi_{j<k}\left(\sigma_{k}-\sigma_{j}\right)=r \times r^{2} \times \ldots \times r^{n-1} \times h_{2}(\boldsymbol{\theta})$ and consequently, we have $\Pi_{j<k}\left|\sigma_{k}-\sigma_{j}\right|=r^{n(n-1) / 2}\left|h_{2}(\boldsymbol{\theta})\right|$. Finally, we have $\left(\sigma_{1} \times \ldots \times \sigma_{n}\right)^{\lambda-1}=r^{n(\lambda-1)} h_{3}(\boldsymbol{\theta})$ and $\left(\sigma_{1}^{2}+\ldots+\sigma_{n}^{2}\right)^{\gamma / 2}=r^{\gamma}$. Substituting these results into Eq. (70) yields

$$
\mathcal{I}_{\varepsilon}=\int_{0}^{\varepsilon} r^{n \lambda-1-\gamma+n(n-1) / 2} d r \int_{\boldsymbol{\theta}} h_{1}(\boldsymbol{\theta})\left|h_{2}(\boldsymbol{\theta})\right| h_{3}(\boldsymbol{\theta}) d \boldsymbol{\theta}
$$

Since $\lambda>0$, we then deduce that

$$
\lambda>\max \left\{0, \frac{\gamma}{n}+\frac{1-n}{2}\right\} \Longrightarrow E\left\{\left\|[\mathbf{A}]^{-1}\right\|_{F}^{\gamma}\right\}<+\infty \quad, \quad \gamma \geq 1 .
$$

Equation (71) shows that if $n=1, n=2$ or $n \geq 3$, then matrix $[\mathbf{A}]^{-1}$ is a second-order random variable $(\gamma=2)$ for $\lambda>2, \lambda>0.5$ or $\lambda>0$ respectively. In addition, it can easily be proved that

$$
\lambda>0 \Longrightarrow E\left\{\|[\mathbf{A}]\|_{F}^{\eta}\right\}<+\infty \quad, \quad \eta>0 .
$$

Equation (72) means that for $\lambda>0$, all the moments of random matrix $[\mathbf{A}]$ exist ( $\eta$ is any positive integer).

\subsection{Monte Carlo simulation of random matrix $[\mathbf{A}]$ when $\lambda$ is a positive integer}

Let us assume that $\lambda$ is a positive integer and let us introduce the positive integer $m_{A}$ such that

$$
m_{A}=n-1+2 \lambda .
$$

Therefore, the characteristic function of random matrix $[\mathbf{A}]$ defined by Eq. (47) can be rewritten as

$$
\Phi_{[\mathbf{A}]}([\Theta])=\left\{\operatorname{det}\left(\left[I_{n}\right]-2 i[C][\Theta]\right)\right\}^{-m_{A} / 2},
$$

in which $[C]=\left(1 / m_{A}\right)[\underline{A}]$. Equation (74) shows that $\Phi_{[\mathbf{A}]}([\Theta])$ corresponds to the characteristic function of a Wishart distribution [70,71] and that random matrix $[\mathbf{A}]$ can be written as $[\mathbf{A}]=\sum_{j=1}^{m_{A}} \mathbf{X}_{j} \mathbf{X}_{j}^{T}$ where $\mathbf{X}_{1}, \ldots, \mathbf{X}_{m_{A}}$ are independent random vectors, each vector $\mathbf{X}_{j}$ being an $\mathbb{R}^{n}$-valued second-order Gaussian random variable, centered and whose covariance matrix is $[C]=E\left\{\mathbf{X}_{j} \mathbf{X}_{j}^{T}\right\}$. Since $[\underline{A}]$ belongs to $\mathbb{M}_{n}^{+}(\mathbb{R})$, the Cholesky factorization of $[\underline{A}]$ yields

$$
[\underline{A}]=\left[L_{A}\right]^{T}\left[L_{A}\right],
$$


in which $\left[L_{A}\right]$ is an upper triangular matrix in $\mathbb{M}_{n}(\mathbb{R})$. Therefore, for all $j$, random vector $\mathbf{X}_{j}$ can be written as $\mathbf{X}_{j}=m_{A}^{-1 / 2}\left[L_{A}\right]^{T} \mathbf{U}_{j}$ in which $\mathbf{U}_{1}, \ldots, \mathbf{U}_{m_{A}}$ are independent random vectors, each vector $\mathbf{U}_{j}$ being an $\mathbb{R}^{n}$-valued second-order Gaussian random variable, centered and whose covariance matrix is $\left[C_{\mathbf{U}_{j}}\right]=E\left\{\mathbf{U}_{j} \mathbf{U}_{j}^{T}\right\}=\left[I_{n}\right]$. Consequently, random matrix $[\mathbf{A}]$ can be written as

$$
[\mathbf{A}]=\frac{1}{m_{A}} \sum_{j=1}^{m_{A}}\left(\left[L_{A}\right]^{T} \mathbf{U}_{j}\right)\left(\left[L_{A}\right]^{T} \mathbf{U}_{j}\right)^{T} .
$$

We then deduce the procedure for the Monte Carlo simulation of random matrix $[\mathbf{A}]$.

1) Simulation of $m_{A} \times n$ independent real-valued normalized Gaussian random variables $\left\{U_{j, k}\right\}_{j, k}$ for $j=$ $1, \ldots, m_{A}$ and $k=1, \ldots, n$ (zero mean value and unit variance).

2) Defining $\left\{\mathbf{U}_{j}\right\}_{k}=U_{j, k}$ and using Eq. (76) yields the simulation of random matrix $[\mathbf{A}]$.

\subsection{Monte Carlo simulation of random matrix $[\mathbf{A}]$ when $\lambda$ is a not an integer}

Let us assume that $\lambda$ is a positive real number (the particular case for which $\lambda$ is a positive integer is presented in Section 3.7). Considering Eq. (75), random matrix $[\mathbf{A}]$ can be written as

$$
[\mathbf{A}]=\left[L_{A}\right]^{T}[\mathbf{G}]\left[L_{A}\right],
$$

in which matrix $[\mathbf{G}]$ is a random variable with values in $\mathbb{M}_{n}^{+}(\mathbb{R})$. From Eqs. (75) and (77), we deduce that the mean value $[\underline{G}]=E\{[\mathbf{G}]\}$ of random matrix $[\mathbf{G}]$ is such that $[\underline{G}]=\left[I_{n}\right]$ in which $\left[I_{n}\right]$ is the unit matrix. The probability density function $p_{[\mathbf{G}]}([G])$ with respect to measure $\widetilde{d} G$ on $\mathbb{M}_{n}^{S}(\mathbb{R})$ of random matrix $[\mathbf{G}]$ with values in $M_{n}^{+}(\mathbb{R})$ is given by Eqs. (48) and (49) in which $[\underline{A}]$ has to be replaced by $\left[I_{n}\right]$. We then have

$$
p_{[\mathbf{G}]}([G])=\mathbb{1}_{M_{n}^{+}(\mathbb{R})}([G]) \times c_{G} \times(\operatorname{det}[G])^{\lambda-1} \times \exp \left(-\frac{(n-1+2 \lambda)}{2} \operatorname{tr}[G]\right),
$$

in which positive constant $c_{G}$ is written as

$$
c_{G}=\frac{(2 \pi)^{-n(n-1) / 4}\left(\frac{n-1+2 \lambda}{2}\right)^{n(n-1+2 \lambda) / 2}}{\left\{\Pi_{\ell=1}^{n} \Gamma\left(\frac{n-\ell+2 \lambda}{2}\right)\right\}} .
$$

Since $[\mathbf{G}]$ is a random matrix with values in $M_{n}^{+}(\mathbb{R})$, the Cholesky factorization allows to write

$$
[\mathbf{G}]=[\mathbf{L}]^{T}[\mathbf{L}] \text { a.s. , }
$$

in which $[\mathbf{L}]$ is an upper triangular random matrix with values in $M_{n}(\mathbb{R})$. Let $[G]$ be a matrix belonging to $M_{n}^{+}(\mathbb{R})$. Therefore, the Cholesky factorization of $[G]$ yields $[G]=[L]^{T}[L]$ in which $[L]$ is an upper triangular matrix such that $\operatorname{det}[L]=\Pi_{j=1}^{n} L_{j j}>0$. For $j \leq k$, let $\widetilde{G}_{j k}$ be such that $\widetilde{G}_{j j}=G_{j j}$ and $\widetilde{G}_{j k}=\sqrt{2} G_{j k}$ if $j<k$, and for $\ell \leq \ell^{\prime}$, let $\widetilde{L}_{\ell \ell^{\prime}}$ be such that $\widetilde{L}_{\ell \ell}=L_{\ell \ell}$ and $\widetilde{L}_{\ell \ell^{\prime}}=\sqrt{2} L_{\ell \ell^{\prime}}$ if $\ell<\ell^{\prime}$. We then deduce that $\widetilde{G}_{j j}=\widetilde{L}_{j j}^{2}+\frac{1}{2} \sum_{\ell<j} \widetilde{L}_{\ell j}^{2}$ and $\widetilde{G}_{j k}=\widetilde{L}_{j j} \widetilde{L}_{j k}+\frac{1}{\sqrt{2}} \sum_{\ell<j} \widetilde{L}_{\ell j} \widetilde{L}_{\ell k}$ for $j<k$. Introducing the mapping $\left\{\widetilde{L}_{\ell \ell^{\prime}}, 1 \leq \ell \leq \ell^{\prime} \leq n\right\} \mapsto\left\{\widetilde{G}_{j k}, 1 \leq\right.$ $j \leq k \leq n\}$ defined by the previous equations and the measures $\widetilde{d} G$ and $\widetilde{d} L$ defined by $\widetilde{d} G=\Pi_{1 \leq j \leq k \leq n} d \widetilde{G}_{j k}=$ $2^{n(n-1) / 4} \Pi_{1 \leq j \leq k \leq n} d G_{j k}$ and $\widetilde{d} L=\Pi_{1 \leq \ell \leq \ell^{\prime} \leq n} d \widetilde{L}_{\ell \ell^{\prime}}=2^{n(n-1) / 4} \Pi_{1 \leq \ell \leq \ell^{\prime} \leq n} d L_{\ell \ell^{\prime}}$, we have (see Appendix A) $\widetilde{d} G=2^{n}\left\{\Pi_{\ell=1}^{n} \widetilde{L}_{\ell \ell}^{n-\ell+1}\right\} \widetilde{d} L$. Let $\widetilde{\mathbb{L}}$ be the vector in $\mathbb{R}^{n(n+1) / 2}$ such that $\widetilde{\mathbb{L}}=\left\{\widetilde{L}_{\ell \ell^{\prime}}, 1 \leq \ell \leq \ell^{\prime} \leq n\right\}$. The corresponding random vector with values in $\mathbb{R}^{n(n+1) / 2}$ associated with random matrix $[\mathbf{L}]$ is denoted as $\widetilde{\mathbf{L}}$. We then have $p_{\tilde{\mathbf{L}}}(\widetilde{\mathbb{L}}) \widetilde{d} L=p_{[\mathbf{G}]}([G]) \widetilde{d} G$, $\operatorname{det}[G]=\sum_{j=1}^{n} \widetilde{L}_{j j}^{2}$ and $\operatorname{tr}[G]=\sum_{j=1}^{n}\left(\widetilde{L}_{j j}^{2}+\frac{1}{2} \sum_{\ell<j} \widetilde{L}_{\ell j}^{2}\right)$. Using Eq. (78) yields $p_{\tilde{\mathbf{L}}}(\widetilde{\mathbb{L}}) \widetilde{d} L=\left\{\Pi_{\ell=1}^{n} p_{\tilde{\mathbf{L}}_{\ell \ell}}\left(\widetilde{L}_{\ell \ell}\right) d \widetilde{L}_{\ell \ell}\right\} \times\left\{\Pi_{\ell<\ell^{\prime}} p_{\tilde{\mathbf{L}}_{\ell \ell^{\prime}}}\left(\widetilde{L}_{\ell \ell^{\prime}}\right) d \widetilde{L}_{\ell \ell^{\prime}}\right\}$ in which $p_{\tilde{\mathbf{L}}_{\ell \ell}}\left(\widetilde{L}_{\ell \ell}\right)$ is the probability density function with respect to $d \widetilde{L}_{\ell \ell}$ of the positive-valued random variable $\widetilde{\mathbf{L}}_{\ell \ell}=\mathbf{L}_{\ell \ell}$, which is written as 
$p_{\tilde{\mathbf{L}}_{\ell \ell}}\left(\widetilde{L}_{\ell \ell}\right)=(n-1+2 \lambda) \widetilde{L}_{\ell \ell} \Gamma_{\ell}\left((n-1+2 \lambda) \widetilde{L}_{\ell \ell}^{2} / 2\right)$ where $\Gamma_{\ell}(y)$ is the gamma probability density function with respect to $d y$ such that

$$
\Gamma_{\ell}(y)=\frac{\mathbb{1}_{[0,+\infty[}(y)}{\Gamma\left(\frac{n-\ell+2 \lambda}{2}\right)} y^{\frac{(n-\ell+2 \lambda)}{2}-1} e^{-y} .
$$

For $\ell<\ell^{\prime}, p_{\tilde{\mathbf{L}}_{\ell \ell^{\prime}}}\left(\widetilde{L}_{\ell \ell^{\prime}}\right)$ is the Gaussian probability density function with respect to $d \widetilde{L}_{\ell \ell^{\prime}}$ of the real-valued random variable $\widetilde{\mathbf{L}}_{\ell \ell^{\prime}}=\sqrt{2} \mathbf{L}_{\ell \ell^{\prime}}$, such that $p_{\tilde{\mathbf{L}}_{\ell \ell^{\prime}}}\left(\widetilde{L}_{\ell \ell^{\prime}}\right)=(2 \pi)^{-1 / 2} \sigma^{-1} \exp \left(-\widetilde{L}_{\ell \ell^{\prime}}^{2} /\left(2 \sigma^{2}\right)\right)$ in which

$$
\sigma=\sqrt{\frac{2}{n-1+2 \lambda}}
$$

We then deduce the procedure for the Monte Carlo simulation of random matrix $[\mathbf{A}]$ :

1) Random variables $\left\{\mathbf{L}_{\ell \ell^{\prime}}, \ell \leq \ell^{\prime}\right\}$ are independent.

2) For $\ell<\ell^{\prime}$, simulation of real-valued Gaussian random variable $\widetilde{\mathbf{L}}_{\ell \ell^{\prime}}$ with zero mean and root mean square given by Eq. (82) and construction of random variable $\mathbf{L}_{\ell \ell^{\prime}}=2^{-1 / 2} \widetilde{\mathbf{L}}_{\ell \ell^{\prime}}$.

3) For $\ell=\ell^{\prime}$, simulation of positive-valued gamma random variable $\mathbf{Y}_{\ell}$ whose probability density function with respect to $d y$ is given by Eq. (81). This random variable is such that its mean value is equal to its variance which is $(n-\ell+2 \lambda) / 2$. We then construct random variable $\widetilde{\mathbf{L}}_{\ell \ell}=\sigma \sqrt{\mathbf{Y}_{\ell}}$ in which $\sigma$ is given by Eq. (82) and we have $\mathbf{L}_{\ell \ell}=\widetilde{\mathbf{L}}_{\ell \ell}$.

4) Calculation of $[\mathbf{G}]=[\mathbf{L}]^{T}[\mathbf{L}]$ and $[\mathbf{A}]=\left[L_{A}\right]^{T}[\mathbf{G}]\left[L_{A}\right]$.

\subsection{Probability model of a set of positive-definite symmetric real random matrices}

Let us consider $\nu$ random matrices $\left[\mathbf{A}_{1}\right], \ldots,\left[\mathbf{A}_{\nu}\right]$ with values in $\mathbb{M}_{n}^{+}(\mathbb{R})$ such that for each $j$ in $\{1, \ldots, \nu\}$, random matrix $\left[\mathbf{A}_{j}\right]$ satisfies Eqs. (32) to (34). This means that only the mean values of the random matrices are known. Applying the maximum entropy principle (see Section 3.3), it can easily be verified that the probability density function $\left(\left[A_{1}\right], \ldots,\left[A_{\nu}\right]\right) \mapsto p_{\left[\mathbf{A}_{1}\right], \ldots,\left[\mathbf{A}_{\nu}\right]}\left(\left[A_{1}\right], \ldots,\left[A_{\nu}\right]\right)$ from $\mathbb{M}_{n}^{+}(\mathbb{R}) \times \ldots \times \mathbb{M}_{n}^{+}(\mathbb{R})$ into $\mathbb{R}^{+}$with respect to the measure (volume element) $\widetilde{d} A_{1} \times \ldots \times \widetilde{d} A_{\nu}$ on $\mathbb{M}_{n}^{S}(\mathbb{R}) \times \ldots \times \mathbb{M}_{n}^{S}(\mathbb{R})$ is written as

$$
p_{\left[\mathbf{A}_{1}\right], \ldots,\left[\mathbf{A}_{\nu}\right]}\left(\left[A_{1}\right], \ldots,\left[A_{\nu}\right]\right)=p_{\left[\mathbf{A}_{1}\right]}\left(\left[A_{1}\right]\right) \times \ldots \times p_{\left[\mathbf{A}_{\nu}\right]}\left(\left[A_{\nu}\right]\right)
$$

which means that $\left[\mathbf{A}_{1}\right], \ldots,\left[\mathbf{A}_{\nu}\right]$ are independent random matrices.

\section{Nonparametric model of random uncertainties for the reduced matrix model}

In this section we complete the construction of the probability model introduced in Section 2 using the developments of Section 3.

\subsection{Probability model of the reduced matrix model}

We apply the results of Section 3 to random matrices $[\mathcal{M}],[\mathcal{D}]$ and $[\mathcal{K}]$ defined in Section 2, for which the available information is described in Section 2.2.2. Parameters $\gamma_{\mathcal{M}}, \gamma_{\mathcal{D}}$ and $\gamma_{\mathcal{K}}$ introduced in Eq. (19) can be chosen as integers such that

$$
\gamma_{\mathcal{M}} \geq 2, \gamma_{\mathcal{D}} \geq 2, \gamma_{\mathcal{K}} \geq 2
$$

In practice, we can take for instance

$$
\gamma_{\mathcal{M}}=\gamma_{\mathcal{D}}=\gamma_{\mathcal{K}}=2
$$

Concerning parameter $\lambda$ allowing the dispersion of the probability model to be controlled (see Section 3.4.8) and concerning its range to satisfy Eq. (19) (see Section 3.6), from Eqs. (19) and (71), we deduce that parameters $\lambda$ for each random matrix $[\mathcal{M}],[\mathcal{D}]$ and $[\mathcal{K}]$, denoted as $\lambda_{\mathcal{M}}, \lambda_{\mathcal{D}}$ and $\lambda_{\mathcal{K}}$ respectively, have to be such that

$$
\lambda_{\mathcal{M}}>\max \left\{0, \frac{\gamma_{\mathcal{M}}}{N}+\frac{1-N}{2}\right\}, \lambda_{\mathcal{D}}>\max \left\{0, \frac{\gamma_{\mathcal{D}}}{N}+\frac{1-N}{2}\right\}, \lambda_{\mathcal{K}}>\max \left\{0, \frac{\gamma_{\mathcal{K}}}{N}+\frac{1-N}{2}\right\}
$$


Parameters $\lambda_{\mathcal{M}}, \lambda_{\mathcal{D}}$ and $\lambda_{\mathcal{K}}$ allow the dispersion of random matrices $[\mathcal{M}],[\mathcal{D}]$ and $[\mathcal{K}]$ to be controlled respectively (see Section 3.4.8). These parameters can be replaced by the parameters $\delta_{\mathcal{M}}, \delta_{\mathcal{D}}$ and $\delta_{\mathcal{K}}$ defined (see Eq. (50)) by

$$
\delta_{\mathcal{M}}=\left\{\frac{E\left\{\|[\mathcal{M}]-[\underline{\mathcal{M}}]\|_{F}^{2}\right\}}{\|[\underline{\mathcal{M}}]\|_{F}^{2}}\right\}^{1 / 2}, \delta_{\mathcal{D}}=\left\{\frac{E\left\{\|[\mathcal{D}]-[\underline{\mathcal{D}}]\|_{F}^{2}\right\}}{\|[\underline{\mathcal{D}}]\|_{F}^{2}}\right\}^{1 / 2}, \delta_{\mathcal{K}}=\left\{\frac{E\left\{\|[\mathcal{K}]-[\underline{\mathcal{K}}]\|_{F}^{2}\right\}}{\|[\underline{\mathcal{K}}]\|_{F}^{2}}\right\}^{1 / 2} .
$$

From Eq. (51), we deduce that

$$
\begin{aligned}
\lambda_{\mathcal{M}} & =\frac{1}{2 \delta_{\mathcal{M}}^{2}}\left(1-\delta_{\mathcal{M}}^{2}(N-1)+\frac{(\operatorname{tr}[\underline{\mathcal{M}}])^{2}}{\operatorname{tr}\left([\underline{\mathcal{M}}]^{2}\right)},\right. \\
\lambda_{\mathcal{D}} & =\frac{1}{2 \delta_{\mathcal{D}}^{2}}\left(1-\delta_{\mathcal{D}}^{2}(N-1)+\frac{(\operatorname{tr}[\underline{\mathcal{D}}])^{2}}{\operatorname{tr}\left([\underline{\mathcal{D}}]^{2}\right)},\right. \\
\lambda_{\mathcal{K}} & =\frac{1}{2 \delta_{\mathcal{K}}^{2}}\left(1-\delta_{\mathcal{K}}^{2}(N-1)+\frac{(\operatorname{tr}[\underline{\mathcal{K}}])^{2}}{\operatorname{tr}\left([\underline{\mathcal{K}}]^{2}\right)}\right) .
\end{aligned}
$$

When Eqs. (88) to (90) are used, $\lambda_{\mathcal{M}}, \lambda_{\mathcal{D}}$ and $\lambda_{\mathcal{K}}$ have to verify Eq. (86). From Section 3.9, we deduce that random matrices $[\mathcal{M}],[\mathcal{D}]$ and $[\mathcal{K}]$ are independent random variables with values in $\mathbb{M}_{N}^{+}(\mathbb{R})$ and from Eqs. (48) and (49), we deduce that the probability density functions $p_{[\mathcal{M}]}, p_{[\mathcal{D}]}$ and $p_{[\mathcal{K}]}$ with respect to the measures (volume elements) $\widetilde{d} \mathcal{M}, \widetilde{d} \mathcal{D}$ and $\widetilde{d K}$ on $M_{N}^{S}(\mathbb{R})$ are given by

$$
\begin{gathered}
p_{[\mathcal{M}]}([\mathcal{M}])=\mathbb{1}_{\mathcal{M}_{N}^{+}(\mathbb{R})}([\mathcal{M}]) \times c_{\mathcal{M}} \times(\operatorname{det}[\mathcal{M}])^{\lambda_{\mathcal{M}}-1} \times \exp \left(-\frac{\left(N-1+2 \lambda_{\mathcal{M}}\right)}{2} \ll[\underline{\mathcal{M}}]^{-1},[\mathcal{M}] \gg\right) \\
p_{[\mathcal{D}]}([\mathcal{D}])=\mathbb{1}_{\mathbb{M}_{N}^{+}(\mathbb{R})}([\mathcal{D}]) \times c_{\mathcal{D}} \times(\operatorname{det}[\mathcal{D}])^{\lambda_{\mathcal{D}}-1} \times \exp \left(-\frac{\left(N-1+2 \lambda_{\mathcal{D}}\right)}{2} \ll[\underline{\mathcal{D}}]^{-1},[\mathcal{D}] \gg\right) \\
p_{[\mathcal{K}]}([\mathcal{K}])=\mathbb{1}_{\mathbb{M}_{N}^{+}(\mathbb{R})}([\mathcal{K}]) \times c_{\mathcal{K}} \times(\operatorname{det}[\mathcal{K}])^{\lambda_{\mathcal{K}}-1} \times \exp \left(-\frac{\left(N-1+2 \lambda_{\mathcal{K}}\right)}{2} \ll[\underline{\mathcal{K}}]^{-1},[\mathcal{K}] \gg\right)
\end{gathered}
$$

in which positive constants $c_{\mathcal{M}}, c_{\mathcal{D}}$ and $c_{\mathcal{K}}$ are written as

$$
\begin{aligned}
& c_{\mathcal{M}}=\frac{(2 \pi)^{-N(N-1) / 4}\left(\left(N-1+2 \lambda_{\mathcal{M}}\right) / 2\right)^{N\left(N-1+2 \lambda_{\mathcal{M}}\right) / 2}}{\left\{\Pi_{\ell=1}^{N} \Gamma\left(\left(N-\ell+2 \lambda_{\mathcal{M}}\right) / 2\right)\right\}(\operatorname{det}[\underline{\mathcal{M}}])^{\left(N-1+2 \lambda_{\mathcal{M}}\right) / 2}} . \\
& c_{\mathcal{D}}= \frac{(2 \pi)^{-N(N-1) / 4}\left(\left(N-1+2 \lambda_{\mathcal{D}}\right) / 2\right)^{N\left(N-1+2 \lambda_{\mathcal{D}}\right) / 2}}{\left\{\Pi_{\ell=1}^{N} \Gamma\left(\left(N-\ell+2 \lambda_{\mathcal{D}}\right) / 2\right)\right\}(\operatorname{det}[\underline{\mathcal{D}}])^{\left(N-1+2 \lambda_{\mathcal{D}}\right) / 2}} . \\
& c_{\mathcal{K}}=\frac{(2 \pi)^{-N(N-1) / 4}\left(\left(N-1+2 \lambda_{\mathcal{K}}\right) / 2\right)^{N\left(N-1+2 \lambda_{\mathcal{K}}\right) / 2}}{\left\{\Pi_{\ell=1}^{N} \Gamma\left(\left(N-\ell+2 \lambda_{\mathcal{K}}\right) / 2\right)\right\}(\operatorname{det}[\underline{\mathcal{K}}])^{\left(N-1+2 \lambda_{\mathcal{K}}\right) / 2}} .
\end{aligned}
$$

Let $\left[h_{N}(\omega ;[\mathcal{M}],[\mathcal{D}],[\mathcal{K}])\right]$ be the function with values in $\mathbb{M}_{m}^{S}(\mathbb{C})$ such that for all $\omega$ in $\mathbb{B}$,

$$
\left[h_{N}(\omega ;[\mathcal{M}],[\mathcal{D}],[\mathcal{K}])\right]=\sum_{\alpha=1}^{N} \sum_{\beta=1}^{N}[\mathcal{T}(\omega ;[\mathcal{M}],[\mathcal{D}],[\mathcal{K}])]_{\alpha \beta} \mathbf{y}_{\alpha} \mathbf{y}_{\beta}^{T}
$$

in which $[\mathcal{T}(\omega ;[\mathcal{M}],[\mathcal{D}],[\mathcal{K}])]$ is the $(N \times N)$ complex symmetric random matrix defined by

$$
[\mathcal{T}(\omega ;[\mathcal{M}],[\mathcal{D}],[\mathcal{K}])]=\left(-\omega^{2}[\mathcal{M}]+i \omega[\mathcal{D}]+[\mathcal{K}]\right)^{-1}
$$

From Eq. (16), we deduce that

$$
\left[\mathbf{H}_{N}(\omega)\right]=\left[h_{N}(\omega ;[\mathcal{M}],[\mathcal{D}],[\mathcal{K}])\right] .
$$

If $\left[H_{N}\right] \mapsto \mathbf{f}\left(\omega ;\left[H_{N}\right]\right)$ is a mapping defined on $\mathbb{M}_{m}^{S}(\mathbb{C})$ with values in $\mathbb{M}_{\nu_{1}, \nu_{2}}(\mathbb{C})$ where $\nu_{1} \geq 1$ and $\nu_{2} \geq 1$, we have

$$
\begin{aligned}
E\left\{\mathbf{f}\left(\omega ;\left[\mathbf{H}_{N}(\omega)\right]\right)\right\} & =\int_{\mathbb{M}_{N}^{+}(\mathbb{R})} \int_{\mathbb{M}_{N}^{+}(\mathbb{R})} \int_{\mathbb{M}_{N}^{+}(\mathbb{R})} \mathbf{f}\left(\omega ;\left[h_{N}(\omega ;[\mathcal{M}],[\mathcal{D}],[\mathcal{K}])\right]\right) \\
& \times p_{[\mathcal{M}]}([\mathcal{M}]) \times p_{[\mathcal{D}]}([\mathcal{D}]) \times p_{[\mathcal{K}]}([\mathcal{K}]) \widetilde{d} \mathcal{M} \widetilde{d} \mathcal{D} \widetilde{d} \mathcal{K}
\end{aligned}
$$




\subsection{Efficient representation of the probability model for the response calculations}

The random responses of the dynamical system with random uncertainties require the calculation of multiple integrals in higher dimension (see Eq. (100)). Two main methods can usually be used to carry out this kind of calculation. The first one corresponds to the perturbation method consisting in performing a $\nu$-order Taylor expansion of function $([\mathcal{M}],[\mathcal{D}],[\mathcal{K}]) \mapsto \mathbf{f}\left(\omega ;\left[h_{N}(\omega ;[\mathcal{M}],[\mathcal{D}],[\mathcal{K}])\right]\right)$ around mean values $([\underline{\mathcal{M}}],[\underline{\mathcal{D}}],[\underline{\mathcal{K}}])$ of random matrices $([\mathcal{M}],[\mathcal{D}],[\mathcal{K}])$. The mathematical expectation can then be calculated using Eqs. (91) to (96). For instance, if function $\mathbf{f}$ is written as $\left.\mathbf{f}\left(\omega ;\left[\mathbf{H}_{N}(\omega)\right]\right)\right\}=[Q(\omega)]\left[\mathbf{H}_{N}(\omega)\right][S(\omega)]\left[\mathbf{H}_{N}(\omega)\right]^{*}[Q(\omega)]^{*}$ in which $[Q(\omega)]$ and $[S(\omega)]$ are given matrices, and if a second-order Taylor expansion of $\left[\mathbf{H}_{N}(\omega)\right]=\left[h_{N}(\omega ;[\mathcal{M}],[\mathcal{D}],[\mathcal{K}])\right]$ with respect to $[\mathcal{M}],[\mathcal{D}]$ and $[\mathcal{K}]$ around their mean values is used, we then have to calculate fourth-order tensor moments of random matrices $[\mathcal{M}],[\mathcal{D}]$ and $[\mathcal{K}]$; this kind of calculation is tricky due to Eqs. (91) to (96) and a more suitable representation of random matrices $[\mathcal{M}],[\mathcal{D}]$ and $[\mathcal{K}]$ has to be used (see below). The second method consists in using the Monte Carlo calculation of multiple integrals with or without variance reduction procedures [73-80]. This method is very efficient if there is a Monte Carlo simulation procedure of random matrices $[\mathcal{M}]$, $[\mathcal{D}]$ and $[\mathcal{K}]$ which is the case for the method presented in Section 3.7, much more efficient than the method presented in Section 3.8. For many applications, the number $N$ is sufficiently high and we can therefore consider $\lambda_{\mathcal{M}}, \lambda_{\mathcal{D}}$ and $\lambda_{\mathcal{K}}$ as positive integers without introducing any significant limitation in the model. The use of the representation introduced in Section 3.7 also makes it easier to develop the perturbation method.

Applying Eq. (76) to random matrices $[\mathcal{M}],[\mathcal{D}]$ and $[\mathcal{K}]$ with values in $\mathbb{M}_{N}^{+}(\mathbb{R})$ and taking into account Eq. (18) yields

$$
\begin{gathered}
{[\mathcal{M}]=\frac{1}{m_{\mathcal{M}}} \sum_{j=1}^{m_{\mathcal{M}}}\left(\left[L_{\mathcal{M}}\right]^{T} \mathbf{U}_{j}\right)\left(\left[L_{\mathcal{M}}\right]^{T} \mathbf{U}_{j}\right)^{T},} \\
{[\mathcal{D}]=\frac{1}{m_{\mathcal{D}}} \sum_{j=1}^{m_{\mathcal{D}}}\left(\left[L_{\mathcal{D}}\right]^{T} \mathbf{V}_{j}\right)\left(\left[L_{\mathcal{D}}\right]^{T} \mathbf{V}_{j}\right)^{T},} \\
{[\mathcal{K}]=\frac{1}{m_{\mathcal{K}}} \sum_{j=1}^{m_{\mathcal{K}}}\left(\left[L_{\mathcal{K}}\right]^{T} \mathbf{W}_{j}\right)\left(\left[L_{\mathcal{K}}\right]^{T} \mathbf{W}_{j}\right)^{T},}
\end{gathered}
$$

in which

$$
m_{\mathcal{M}}=N-1+2 \lambda_{\mathcal{M}} \quad, \quad m_{\mathcal{D}}=N-1+2 \lambda_{\mathcal{D}} \quad, \quad m_{\mathcal{K}}=N-1+2 \lambda_{\mathcal{K}},
$$

where $\lambda_{\mathcal{M}}, \lambda_{\mathcal{D}}$ and $\lambda_{\mathcal{K}}$ are positive integers verifying Eq. (86) and given by Eqs. (88) to (90), and where [ $\left.L_{\mathcal{M}}\right]$, $\left[L_{\mathcal{D}}\right]$ and $\left[L_{\mathcal{K}}\right]$ are upper triangular matrices in $M_{N}(\mathbb{R})$ corresponding to the Cholesky factorization of matrices $[\underline{\mathcal{M}}],[\underline{\mathcal{D}}]$ and $[\underline{\mathcal{K}}]$ in $\mathbb{M}_{N}^{+}(\mathbb{R})$ such that

$$
[\underline{\mathcal{M}}]=\left[L_{\mathcal{M}}\right]^{T}\left[L_{\mathcal{M}}\right] \quad, \quad[\underline{\mathcal{D}}]=\left[L_{\mathcal{D}}\right]^{T}\left[L_{\mathcal{D}}\right] \quad, \quad[\underline{\mathcal{K}}]=\left[L_{\mathcal{K}}\right]^{T}\left[L_{\mathcal{K}}\right] .
$$

The set of all the components of random vectors $\mathbf{U}_{1}, \ldots, \mathbf{U}_{m_{\mathcal{M}}}$ with values in $\mathbb{R}^{N}$ and of random vectors $\mathbf{V}_{1}, \ldots, \mathbf{V}_{m_{\mathcal{D}}}$ and $\mathbf{W}_{1}, \ldots, \mathbf{W}_{m_{\mathcal{K}}}$ with values in $\mathbb{R}^{N}$ is constituted of $m_{\mathcal{M}} \times N+m_{\mathcal{D}} \times N+m_{\mathcal{K}} \times N$ independent random variables, each of which is a real-valued second-order normalized Gaussian random variable (zero mean value and unit variance).

For an efficient computation, Eqs. (101) to (103) can be rewritten as

$$
[\mathcal{M}]=\frac{1}{m_{\mathcal{M}}} \sum_{j=1}^{m_{\mathcal{M}}}\left[\mathcal{M}^{j}\right] \quad, \quad[\mathcal{D}]=\frac{1}{m_{\mathcal{D}}} \sum_{j=1}^{m_{\mathcal{D}}}\left[\mathcal{D}^{j}\right] \quad, \quad[\mathcal{K}]=\frac{1}{m_{\mathcal{K}}} \sum_{j=1}^{m_{\mathcal{K}}}\left[\mathcal{K}^{j}\right]
$$

in which random matrices $\left[\mathcal{M}^{j}\right],\left[\mathcal{D}^{j}\right]$ and $\left[\mathcal{K}^{j}\right]$ with values in $\mathbb{M}_{N}^{+}(\mathbb{R})$ are such that

$$
\left[\mathcal{M}^{j}\right]=\mathcal{U}_{j} \mathcal{U}_{j}^{T} \quad, \quad\left[\mathcal{D}^{j}\right]=\mathcal{V}_{j} \mathcal{V}_{j}^{T} \quad, \quad\left[\mathcal{K}^{j}\right]=\mathcal{W}_{j} \mathcal{W}_{j}^{T}
$$

in which for $j$ fixed and for $\alpha=1, \ldots, N$, we have

$$
\mathcal{U}_{j}=\left[L_{\mathcal{M}}\right]^{T} \mathbf{U}_{j} \quad, \quad \mathcal{V}_{j}=\left[L_{\mathcal{D}}\right]^{T} \mathbf{V}_{j} \quad, \quad \mathcal{W}_{j}=\left[L_{\mathcal{K}}\right]^{T} \mathbf{W}_{j}
$$




\section{Simple example}

We consider the "mean finite element model" defined by Eq. (2) for which $m=50$ and $m_{\text {rig }}=0$, frequency band $\mathbb{B}$ of analysis is $[1,50] \mathrm{Hz}$ and the mean mass, damping and stiffness matrices are such that $[\underline{M}]_{j k}=\delta_{j k}$, $[\underline{D}]_{j k}=2 \xi \omega_{j} \delta_{j k}$ and $[\underline{K}]_{j k}=\omega_{j}^{2} \delta_{j k}$ with $0<\omega_{1}<\omega_{2}<\ldots<\omega_{m}$ and $\xi=0.01$. Eigenfrequencies $\omega_{1}, \ldots, \omega_{m}$ correspond to independent samples of a uniformly distributed random variable over the frequency band $[5,150] \mathrm{Hz}$ and are such that $\omega_{1}, \omega_{20}, \omega_{21}, \omega_{25}$ and $\omega_{50}$ are $5.04 \mathrm{~Hz}, 47.87 \mathrm{~Hz}, 50.71 \mathrm{~Hz}, 60.28 \mathrm{~Hz}$ and 137.38 $\mathrm{Hz}$ respectively. It should be noted that, for this simple example, the "mean finite element model" can be viewed as a "generalized mean finite element model" which corresponds to the projection on the first structural modes of a "mean finite element model" which is not described here and for which the damping operator is diagonalized by the structural modes. Figure 2 shows the graph of the acceleration spectrum expressed in $\mathrm{dB}$ and defined by

$$
\omega \mapsto \underline{d B}(\omega)=10 \log _{10}\left(\omega^{4}\|[\underline{h}(\omega)]\|_{F}^{2}\right) \quad,
$$

in which $[\underline{h}(\omega)]$ is defined by Eq. (3). We consider the reduced matrix model defined in Section 4 with $N=25$ and three cases for the dispersion of the generalized mass, damping and stiffness random matrices (see Eqs. (87) to $(90))$,

Case 1: $\delta_{M}=0.2, \delta_{D}=0.2$ and $\delta_{K}=0.2$ corresponding to $\lambda_{M}=312, \lambda_{D}=258$ and $\lambda_{K}=204$.

Case 2: $\delta_{M}=0.1, \delta_{D}=0.1$ and $\delta_{K}=0.1$ corresponding to $\lambda_{M}=1287, \lambda_{D}=1071$ and $\lambda_{K}=855$.

Case 3: $\delta_{M}=0.03, \delta_{D}=0.03$ and $\delta_{K}=0.03$ corresponding to $\lambda_{M}=14432, \lambda_{D}=12026$ and $\lambda_{K}=9630$.

Cases 1, 2 and 3 correspond to high, medium and low dispersion respectively. We are interested in the random acceleration spectrum expressed in $\mathrm{dB}$ and defined by

$$
\omega \mapsto \mathbf{d B}(\omega)=10 \log _{10}\left(\omega^{4}\left\|\left[\mathbf{H}_{N}(\omega)\right]\right\|_{F}^{2}\right) \quad,
$$

in which random matrix $\left[\mathbf{H}_{N}(\omega)\right]$ is defined by Eq. (99). For each case, Monte Carlo numerical simulation is carried out with $n_{S}$ samples, denoted as $\theta_{1}, \ldots, \theta_{n_{S}}$, for which the samples $\omega \mapsto \mathbf{d B}\left(\omega ; \theta_{1}\right), \ldots, \omega \mapsto \mathbf{d B}\left(\omega ; \theta_{n_{S}}\right)$ are numerically calculated on frequency band $[1,50] \mathrm{Hz}$ with a sampling frequency step $0.1 \mathrm{~Hz}$. For $\omega$ fixed, the mean value of random variable $\mathbf{d B}(\omega)$ is estimated by

$$
E\{\mathbf{d B}(\omega)\} \simeq \frac{1}{n_{S}} \sum_{j=1}^{n_{S}} \mathbf{d B}\left(\omega ; \theta_{j}\right) .
$$

Finally, we introduce the functions $\omega \mapsto d B_{\max }(\omega ; \boldsymbol{\theta})$ and $\omega \mapsto d B_{\min }(\omega ; \boldsymbol{\theta})$ defined by

$$
d B_{\max }(\omega ; \boldsymbol{\theta})=\max _{j=1, \ldots, n_{S}} \mathbf{d B}\left(\omega ; \theta_{j}\right) \quad, \quad d B_{\min }(\omega ; \boldsymbol{\theta})=\min _{j=1, \ldots, n_{S}} \mathbf{d B}\left(\omega ; \theta_{j}\right)
$$

in which $\boldsymbol{\theta}=\left(\theta_{1}, \ldots, \theta_{n_{S}}\right)$. Figures 3,4 and 5 are related to cases 1,2 and 3 for $n_{S}=100$ and Figures 6 , 7 and 8 are related to cases 1,2 and 3 for $n_{S}=1000$. Each figure shows four graphs: the graph of function $\omega \mapsto \underline{d B}(\omega)$ (thin solid line) defined by Eq. (109) related of the mean reduced matrix model, the graph of function $\omega \mapsto \bar{E}\{\mathbf{d B}(\omega)\}$ (dashed line) estimated by Eq. (111) and finally, the graphs of functions $\omega \mapsto d B_{\max }(\omega ; \boldsymbol{\theta})$ and $\omega \mapsto d B_{\min }(\omega ; \boldsymbol{\theta})$ (thick solid lines) defined by Eq. (112).

\section{Conclusions}

We have presented the theoretical basis of a new method allowing the random uncertainties to be modeled for reduced matrix models in linear structural dynamics. The information used does not require the description of the local parameters of the mechanical model. The probability model is deduced from the use of the entropy optimization principle whose available information is constituted of the fundamental algebraic properties related to the generalized mass, damping and stiffness matrices which have to be positive-definite symmetric matrices, and the knowledge of these matrices for the mean reduced matrix model. An explicit construction and representation of the probability model have been obtained and are very well adapted to algebraic calculus and to Monte Carlo numerical simulation in order to compute the responses of the dynamical system. 


\section{Appendix A}

This appendix concerns the calculation of the Jacobian matrix of transformation $[L] \mapsto[G]=[L]^{T}[L]$ of symmetric positive-definite matrix $[G]$. Let $[G]$ be a positive-definite symmetric $(n \times n)$ real matrix (i.e. $\left.[G] \in \mathbb{M}_{n}^{+}(\mathbb{R})\right)$ and let $\widetilde{G}_{j k}$ be such that $\widetilde{G}_{j j}=G_{j j}$ and $\widetilde{G}_{j k}=\sqrt{2} G_{j k}$ if $j<k$. Consequently, there exists an upper triangular matrix $[L] \in \mathbb{M}_{n}(\mathbb{R})$ such that $[G]=[L]^{T}[L]$ with $[L]_{\ell \ell}>0$. For $\ell \leq \ell^{\prime}$, we define $\widetilde{L}_{\ell \ell^{\prime}}$ such that $\widetilde{L}_{\ell \ell}=L_{\ell \ell}$ and $\widetilde{L}_{\ell \ell^{\prime}}=\sqrt{2} L_{\ell \ell^{\prime}}$ if $\ell<\ell^{\prime}$. A simple calculation yields $\widetilde{G}_{j j}=\widetilde{L}_{j j}^{2}+\frac{1}{2} \sum_{\ell<j} \widetilde{L}_{\ell j}^{2}$ and $\widetilde{G}_{j k}=$ $\widetilde{L}_{j j} \widetilde{L}_{j k}+\frac{1}{\sqrt{2}} \sum_{\ell<j} \widetilde{L}_{\ell j} \widetilde{L}_{\ell k}$ if $j<k$. Let $[\widetilde{J}]$ be the Jacobian matrix of the mapping $\left\{\widetilde{L}_{\ell \ell^{\prime}}, 1 \leq \ell \leq \ell^{\prime} \leq n\right\} \mapsto$ $\left\{\widetilde{G}_{j k}, 1 \leq j \leq k \leq n\right\}$ defined by the previous equations. For $1 \leq j \leq k \leq n$ and $1 \leq \ell \leq \ell^{\prime} \leq n$, we have $[\widetilde{J}]_{j k, \ell \ell^{\prime}}=\partial \widetilde{G}_{j k} / \partial \widetilde{L}_{\ell \ell^{\prime}}$. We then deduce that $\operatorname{det}[\widetilde{J}]=2^{n}\left\{\Pi_{\ell=1}^{n} \widetilde{L}_{\ell \ell}^{n-\ell+1}\right\}$ and therefore, $\widetilde{d} G=|\operatorname{det}[\widetilde{J}]| \widetilde{d} L$ in which $\widetilde{d} G$ is the measure defined by $\widetilde{d} G=\Pi_{1 \leq j \leq k \leq n} d \widetilde{G}_{j k}=2^{n(n-1) / 4} \Pi_{1 \leq j \leq k \leq n} d G_{j k}$ and $\widetilde{d} L$ is the measure defined by $\widetilde{d} L=\Pi_{1 \leq \ell \leq \ell^{\prime} \leq n} d \widetilde{L}_{\ell \ell^{\prime}}=2^{n(n-1) / 4} \Pi_{1 \leq \ell \leq \ell^{\prime} \leq n} d L_{\ell \ell^{\prime}}$. Since $\widetilde{L}_{\ell \ell}>0$, we deduce that $\widetilde{d} G=2^{n}\left\{\Pi_{\ell=1}^{n} \widetilde{L}_{\ell \ell}^{n-\ell+1}\right\} \widetilde{d} L$.

\section{Appendix B}

This appendix concerns the transformation from rectangular to polar coordinates in $\mathbb{R}^{n}$. Let $\boldsymbol{\sigma}=\left(\sigma_{1}, \ldots, \sigma_{n}\right)$ be the rectangular coordinates in $\mathbb{R}^{n}$ and $(r, \boldsymbol{\theta})$ be the polar coordinates such that $\boldsymbol{\theta}=\left(\theta_{1}, \ldots, \theta_{n-1}\right)$. The transformation is defined by $\sigma_{1}=r \sin \theta_{1}, \sigma_{2}=r \cos \theta_{1} \sin \theta_{2}, \ldots, \sigma_{n-1}=r \cos \theta_{1} \cos \theta_{2} \ldots \cos \theta_{n-2} \sin \theta_{n-1}$ and $\sigma_{n}=$ $r \cos \theta_{1} \cos \theta_{2} \ldots \cos \theta_{n-2} \cos \theta_{n-1}$, in which $-\pi / 2<\theta_{j} \leq \pi / 2$ for $j=1, \ldots, n-2$ and $-\pi<\theta_{n-1} \leq \pi$. We have $\sum_{j=1}^{n} \sigma_{j}^{2}=r^{2}$ and $d \boldsymbol{\sigma}=r^{n-1} h_{1}(\boldsymbol{\theta}) d r d \boldsymbol{\theta}$ in which $h_{1}(\boldsymbol{\theta})=\left|\cos ^{n-2} \theta_{1} \cos ^{n-3} \theta_{3} \ldots \cos \theta_{n-2}\right|$.

\section{References}

[ 1] Bathe, K. J. and Wilson, E. L., Numerical Methods in Finite Element Analysis. Prentice-Hall, New York, 1976.

[ 2] Meirovitch, L., Computational Methods in Structural Dynamics. Sijthoff and Noordhoff, The Netherlands, 1980.

[ 3] Zienkiewicz, O. C. and Taylor, R. L., The Finite Element Method (4th edition). McGraw-Hill, New York, 1989 (vol.1, 1989 and vol. 2, 1991).

[ 4] Argyris, J. and Mlejnek, H. P., Dynamics of Structures. North-Holland, Amsterdam, 1991.

[ 5] Cheung, Y. K. and Leung, A. Y. T., Finite Element Methods in Dynamics. Kluwer Academic Pub, 1992.

[ 6] Ohayon, R. and Soize, C., Structural Acoustics and Vibration. Academic Press, San Diego, London, 1998.

[ 7] Soize, C., Reduced models in medium frequency range for general dissipative structural-dynamics systems. European Journal of Mechanics, A/Solids, 1998, 17(4), 657-685.

[ 8] Soong, T. T., Random Differential Equations in Science and Engineering. Academic Press, New York, 1973.

[ 9] Chen, P. C. and Soroka, W. W., Multi-degree dynamic response of a system with statistical properties. Journal of Sound and Vibration, 1973, 37(4), 547-556.

[ 10] Prasthofer P. H. and Beadle, C. W., Dynamic response of structures with statistical uncertainties in their stiffness. Journal of Sound and Vibration, 1975, 42(4), 477-493.

[ 11] Haug, E. J., Choi, K. K. and Komkov V., Design Sensitivity Analysis of Structural Systems. Academic Press, 1986.

[ 12] Bergman, L. A. and Spencer Jr, B. F., First passage time for linear systems with stochastic coefficients. Probabilistic Engineering Mechanics, 1987, 2(1), 46-53.

[ 13] Ibrahim, R. A., Structural dynamics with parameter uncertainties. Applied Mechanics Reviews, 1987, 40(3), 309-328.

[ 14] Bucher, C. GT. and Brenner, C. E., Stochastic response of uncertain systems. Archive of Applied Mechanics, 1992, 62, 507-516.

[ 15] Spanos, P. D. and Zeldin, B. A., Galerkin sampling method for stochastic mechanics problems. Journal of Engineering Mechanics, 1994, 120(5), 1091-1106.

[ 16] Katafygiotis, L. S. and Beck, J. L., A very efficient moment calculation method for uncertain linear dynamics. Probabilistic Engineering Mechanics, 1995, 10(2), 117-128.

[ 17] Singh, R. and Lee, C., Frequency response of linear systems with parameter uncertainties. Journal of Sound and Vibration, 1993, 168(1), 71-92. 
[ 18] Iwan, W. D. and Jensen, H., On the dynamical response of continuous systems including model uncertainty. Transactions of ASME, 1993, 60, 484-490.

[ 19] Lee, C. and Singh, R., Analysis of discrete vibratory systems with parameter uncertainties. Part I: Eigensolution. Journal of Sound and Vibration, 1994, 174(3), 379-394.

[ 20] Lee, C. and Singh, R., Analysis of discrete vibratory systems with parameter uncertainties. Part II: Impulse response. Journal of Sound and Vibration, 1994, 174(3), 395-412.

[ 21] Lin, Y.K. and Cai, G. Q., Probabilistic Structural Dynamics. McGraw-Hill, New York, 1995.

[ 22] Papadimitriou, C., Katafygiotis, L. S. and Beck, J. L., Approximate analysis of response variability of uncertain linear systems. Probabilistic Engineering Mechanics, 1995, 10(4), 251-264.

[ 23] Collins, J. D. and Thomson, W. T., The eigenvalue problem for structural systems with statistical properties. AIAA Journal, 1969, 7(4), 642-648.

[ 24] Shinozuka, M. and Astill, C. J., Random eigenvalue problems in structural analysis. AIAA Journal, 1972, 10(4), 456-462.

[ 25] Shinozuka, M. and Lenoe, E., A probabilistic model for spatial distribution of material properties. Engineering Fracture Mechanics, 1976, 8(1), 217-227.

[ 26] Kotulski, Z. and Sobczyk, K., Effects of parameter uncertainty on the response of vibratory systems to random excitation. Journal of Sound and Vibration, 1987, 119(1), 159-171.

[ 27] Poirion, F., Effect of structural uncertainties on flutter analysis. Recherche Aerospatiale (English edition), 1992, 6, 31-41.

[ 28] Jensen, H. and Iwan, W. D., Response of systems with uncertain parameters to stochastic excitation. Journal of Engineering Mechanics, 1992, 118(5), 1012-1025.

[ 29] Manohar, C. S., Adhikari, S., Dynamic stiffness of randomly parametered beams. Probabilistic Engineering Mechanics, 1998, 13(1), 39-51.

[ 30] Micaletti, R. C., Cakmak, A. S., Nielsen, S. R. K. and Koyluglu, H. U., A solution method for linear and geometrically nonlinear MDOF systems with random properties subject to random excitation. Probabilistic Engineering Mechanics, 1998, 13(2), 85-95.

[ 31] Vanmarcke, E. and Grigoriu, M., Stochastic finite element analysis of simple beams. Journal of Engineering Mechanics, 1983, 109(5), 1203-1214.

[ 32] Liu, W. K., Belytschko, T. and Mani, A., Random field finite elements. International Journal of Numerical Methods in Engineering, 1986, 23, 1832-1845.

[ 33] Shinozuka, M. and Deodatis, G., Response variability of stochastic finite element systems. Journal of Engineering Mechanics, 1988, 114(3), 499-519.

[ 34] Spanos, P. D. and Ghanem, R. G., Stochastic finite element expansion for random media. ASCE Journal of Engineering Mechanics, 1989, 115(5), 1035-1053.

[ 35] Ghanem, R. G. and Spanos, P. D., Stochastic Finite Elements: A Spectral Approach. Springer-Verlag, New York, 1991.

[ 36] Kleiber M., Tran, D. H. and Hien, T. D., The Stochastic Finite Element Method. John Wiley and Sons, 1992.

[ 37] Ditlevsen, O. and Tarp-Johansen, N. J., Choice of input fields in stochastic finite elements. Probabilistic Engineering Mechanics, 1998, 14(1-2), 63-72.

[ 38] Papadopoulos, V. and Papadrakakis, M., Stochastic finite element-based reliability analysis. Probabilistic Engineering Mechanics, 1998, 13(1), 53-65.

[ 39] Lin, Y.K., Probabilistic Theory of Structural Dynamics. McGraw-Hill, New York, 1967 (Reprint R.E., Krieger, Melbourne, Fla., 1976).

[ 40] Elishakoff, I., Probabilistic Methods in the Theory of Structures. Wiley, New York, 1983.

[ 41] Ibrahim, R. A., Parametric Random Vibration. John Wiley and Sons, New York, 1985.

[ 42] Kree, P. and Soize, C., Mathematics of Random Phenomena. Reidel, Dordrecht, 1986.

[ 43] Roberts, J.B. and Spanos, P.D., Random Vibration and Statistical Linearization. John Wiley and Sons, New York, 1990.

[ 44] Sobczyk, K., Stochastic Differential Equations with Applications to Physics and Engineering. Kluewer, Dordrecht, Boston, 1991. 
[ 45] Soong, T. T. and Grigoriu, M. Random Vibration of Mechanical and Structural Systems. P T R Prentice Hall, Englewood Cliffs, New Jersey, 1993.

[ 46] Soize, C., The Fokker-Planck Equation for Stochastic Dynamical Systems and its Explicit Steady State Solutions. World Scientific, Singapore, 1994.

[ 47] Sundararajan, C. (Editor), Probabilistic Structural Mechanics Handbook. Chapman and Hall, 1995.

[ 48] Sarkani, S. and Lutes, L. D., Stochastic Analysis of Structural and Mechanical Vibrations. Prentice-Hall, New York, 1996.

[ 49] Schueller, G. I. (editor), A state-of-the-art report on computational stochastic mechanics. Probabilistic Engineering Mechanics, 1997, 12(4), 197-321.

[ 50] Soize, C., Sur le calcul des densités spectrales des réponses stationnaires pour des systèmes dynamiques stochastiques non-linéaires. Rencontres Scientifiques du Cinquantenaire, Contrôle Actif Vibro-acoustique et Dynamique Stochastique. Publications du LMA-CNRS, ISSN 0750-7356, 127, 297-344, 1991.

[ 51] Bouc, R., The power spectral density of response for a strongly nonlinear random oscillator. Journal of Sound and Vibration, 1994, 175(3), 317-331.

[ 52] Bellizzi, S. and Bouc, R., Analysis of multi-degrees of freedom strongly non-linear mechanical systems with random input. Part II: Equivalent linear system with random matrices and power spectral density matrix. Probabilistic Engineering Mechanics, 1999, 14, 245-256.

[ 53] Soize, C., Stochastic linearization method with random parameters for SDOF nonlinear dynamical systems: prediction and identification procedures. Probabilistic Engineering Mechanics, 1995, 10(3), 143-152.

[ 54] Bellizi, S. and Bouc, R., Spectral response of asymmetrical random oscillators. Probabilistic Engineering Mechanics, 1996, 11(1), 51-59.

[ 55] Bouc, R. and Defilippi, M., Multimodal nonlinear spectral response of a beam with impact under random input. Probabilistic Engineering Mechanics, 1997, 12(3), 163-170.

[ 56] Soize, C. and Lefur, O., Modal identification of weakly nonlinear multidimensional dynamical systems using a stochastic linearization method with random coefficients. Journal of Mechanical System and Signal Processing, 1997, 11(1), 37-40.

[ 57] Soize, C., Probabilistic structural modeling in linear dynamic analysis of complex mechanical systems, I Theoretical elements. La Recherche Aérospatiale, (English edition), 1986, 5, $23-48$.

[ 58] Soize, C., A model and numerical method in the medium frequency range for vibroacoustic predictions using theory of structural fuzzy. J. Acoust. Soc. Am., 1993, 94(2), Pt 1, 849-866.

[ 59] Mead, L. R. and Papanicolau, N., Maximum entropy in the moment problem. J. Math. Phys., 1984, 25(8), 2404-2417.

[ 60] Rosenblueth, E., Karmeshu and Hong, H. P., Maximum entropy and discretization of probability distributions. Probabilistic Engineering Mechanics, 1987, 2(2), 58-63.

[ 61] Tagliani, A., Principle of maximum entropy and probability distributions: definition of applicability field. Probabilistic Engineering Mechanics, 1989, 4(2), 99-104.

[ 62] Sobczyk, K. and Trebicki, J., Maximum entropy principle in stochastic dynamics. Probabilistic Engineering Mechanics, 1990, 5(3), 102-110.

[ 63] Jumarie, G., Solution of the multivariate Fokker-Planck equation by using a maximum path entropy principle. $J$. Math. Phys., 1990, 31(10), 2389-2392.

[ 64] Trebicki, J. and Sobczyk, K. J., Maximum entropy principle and non-stationary distributions of stochastic systems Probabilistic Engineering Mechanics, 1996, 11(3), 169-178.

[ 65] Shannon, C. E., A mathematical theory of communication. Bell System Tech. J., 1948, 27, 379-423 and 623-659.

[ 66] Jaynes, E. T., Information theory and statistical mechanics. Physical Review, 1957, 106(4), 620-630 and 108(2), 171-190.

[ 67] Kapur, J. N. and Kesavan, H. K., Entropy Optimization Principles with Applications. Academic Press, San Diego, 1992.

[ 68] Fougeaud, C. and Fuchs, A., Statistique. Dunod, Paris, 1967 (2nd edition 1972).

[ 69] Arnold, V., Mathematical Methods of Classical Mechanics. Springer-Verlag, New York, 1978.

[ 70] Anderson, T. W., Introduction to Multivariate Statistical Analysis. John Wiley and Sons, 1958.

[ 71] Saporta, G., Probabilités Analyse des Données et Statistique. Editions Technip, Paris, 1990. 
[ 72] Mehta, M. L., Random Matrices. Revised and Enlarged Second Edition, Academic Press, 1991.

[ 73] Hammersley, J. M. and Handscomb, D. C., Monte Carlo Methods. Chapman and Hall, 1964.

[ 74] Cochran, W. G., Sampling Techniques. John Wiley and Sons, 1977.

[ 75] Rubinstein, R. Y., Simulation and the Monte Carlo Method. John Wiley and Sons, New York, 1981.

[ 76] Devroye, L., Non Uniform Random Variate Generation. Springer-Verlag, New York, 1986.

[ 77] Kalos, M. H. and Whitlock, P. A., Monte Carlo Methods, Volume 1: Basics. John Wiley and Sons, 1986.

[ 78] Bratley, P., Fox, B. L. and Schrage, E. L., A Guide to Simulation. Springer-Verlag, New York, 2nd edition, 1987.

[ 79] MacKeown, P. K., Stochastic Simulation in Physics. Springer-Verlag, Singapore, 1997.

[ 80] Lapeyre, B., Pardoux, E. and Sentis, R., Méthodes de Monte-Carlo pour les Equations de Transport et de Diffusion. Springer-Verlag, Berlin, 1998. 


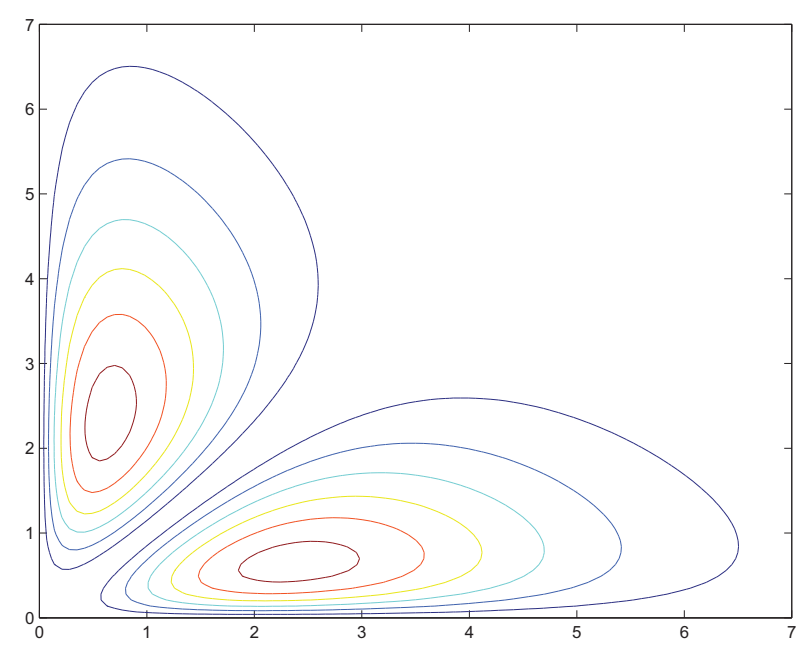

Fig. 1. Contour plot of function $\left(\sigma_{1}, \sigma_{2}\right) \mapsto c_{\Sigma}^{-1} p_{[\Sigma]}\left(\sigma_{1}, \sigma_{2}\right)$

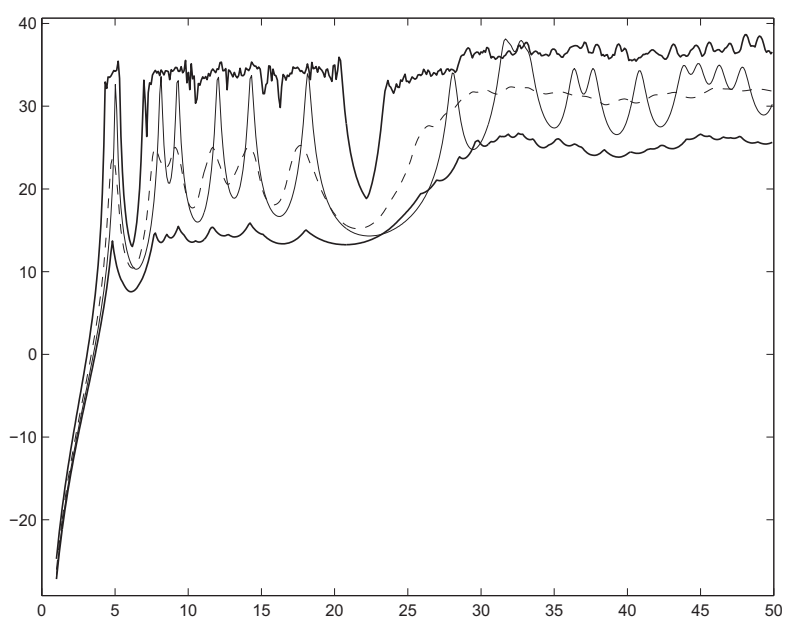

Fig. 3. Acceleration spectrum in $\mathrm{dB}$ for case 1 and $n_{S}=100$

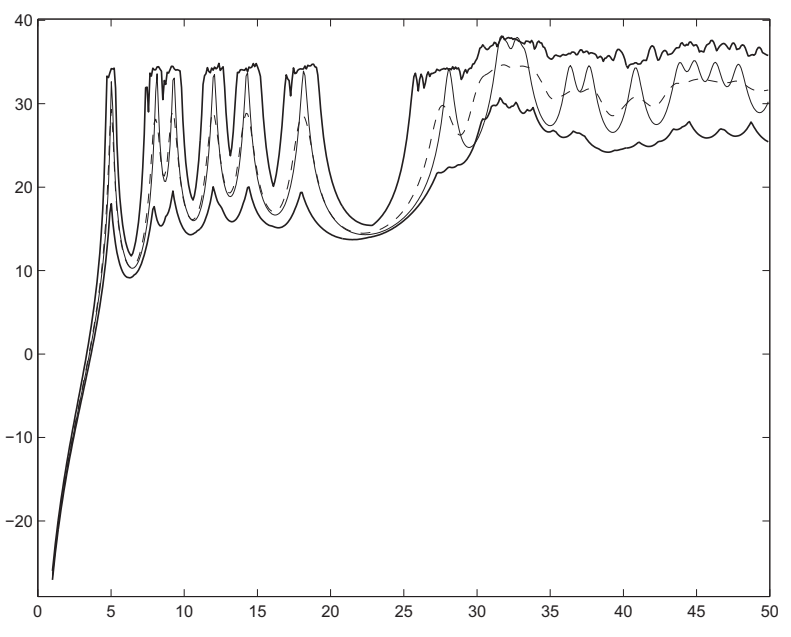

Fig. 4. Acceleration spectrum in $\mathrm{dB}$ for case 2 and $n_{S}=100$

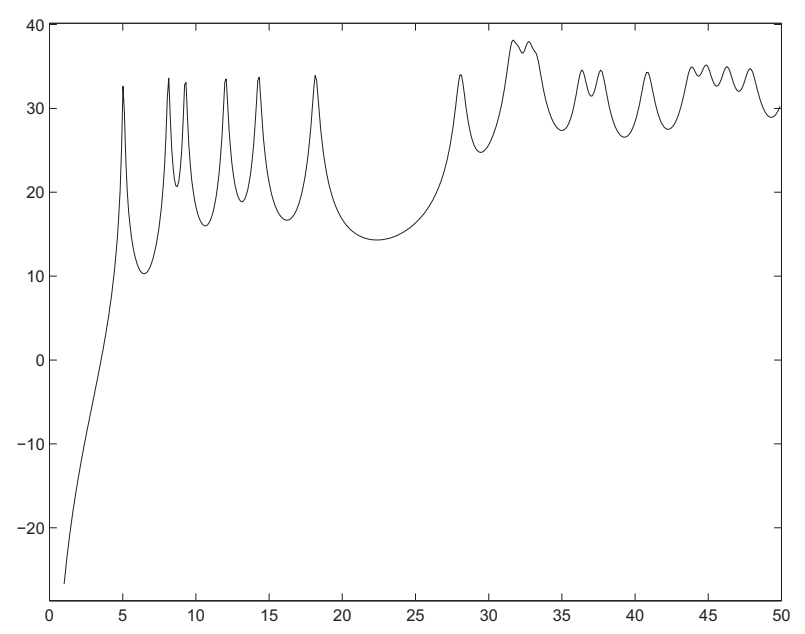

Fig. 2. Acceleration spectrum in $\mathrm{dB}$ of the "mean finite element model"

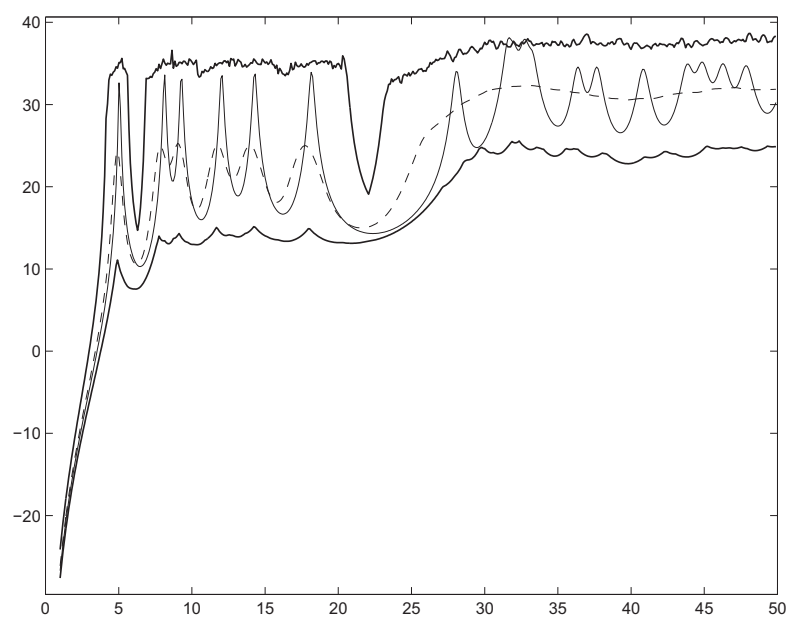

Fig. 6. Acceleration spectrum in $\mathrm{dB}$ for case 1 and $n_{S}=1000$

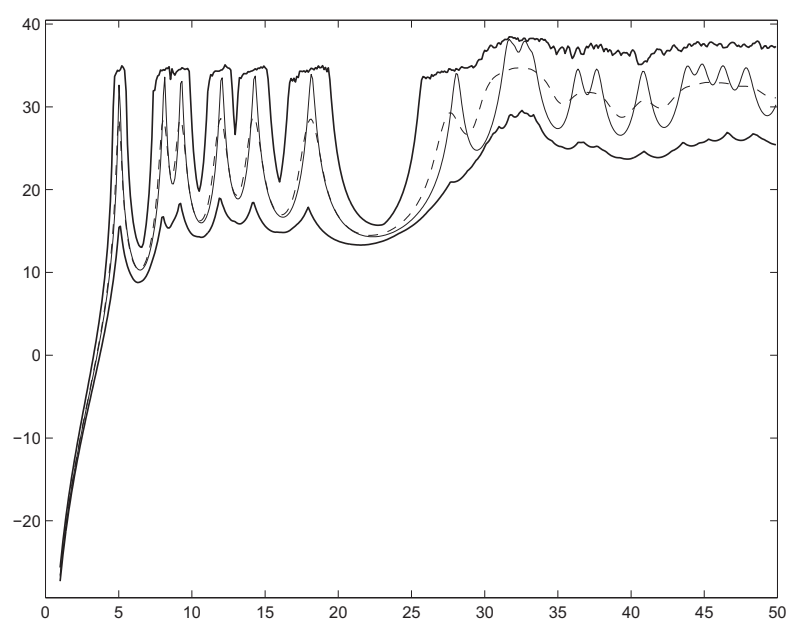

Fig. 7. Acceleration spectrum in $\mathrm{dB}$ for case 2 and $n_{S}=1000$

Proba. Eng. Mech. 


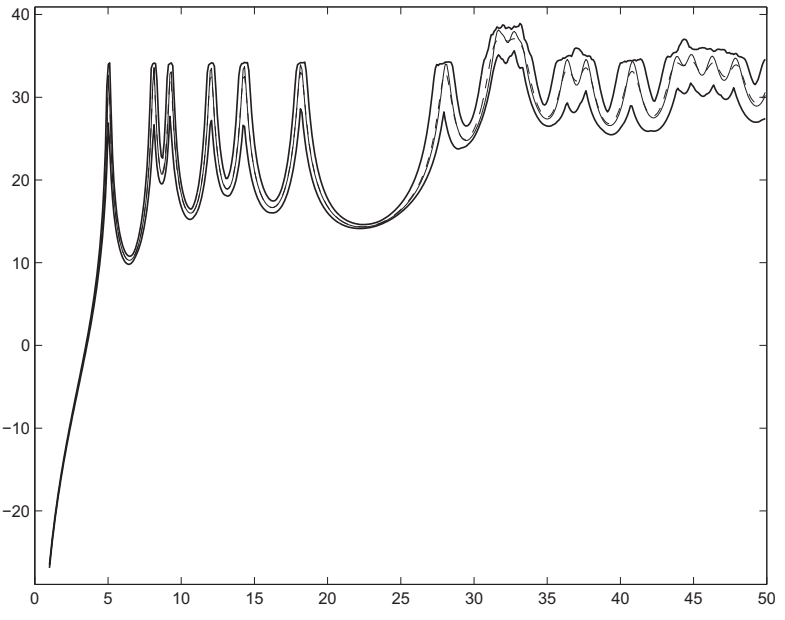

Fig. 5. Acceleration spectrum in $\mathrm{dB}$ for case 3 and $n_{S}=100$

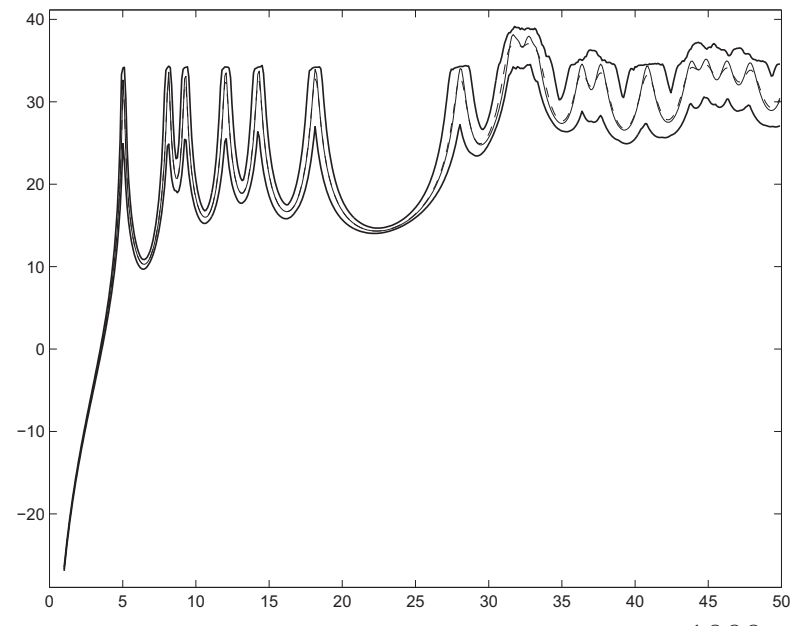

Fig. 8. Acceleration spectrum in $\mathrm{dB}$ for case 3 and $n_{S}=1000$
Proba. Eng. Mech.
C. Soize-October 1999 